\begin{tabular}{|c|c|}
\hline Title & Strongly nonlinear waves and streaming in the near field of a circular piston \\
\hline Author(s) & Y ano, Takeru; Inoue, Y oshinori \\
\hline Citation & $\begin{array}{l}\text { The Journal of the A coustical Society of A merica, 99(6), 3353 } 3372 \\
\text { https://doi.org/10.1121/1.414892 }\end{array}$ \\
\hline Issue Date & 1996-06-01 \\
\hline Doc URL & http:/hdl.handle.net/2115/14913 \\
\hline Rights & $\begin{array}{l}\text { Copyright 1996, A coustical Society of A merica. This article may be downloaded for personal use only. A ny other use } \\
\text { requires prior permission of the author and the A coustical Society of A merica. }\end{array}$ \\
\hline Type & article \\
\hline File Information & JA SA 99-6.pdf \\
\hline
\end{tabular}

Instructions for use 


\title{
Strongly nonlinear waves and streaming in the near field of a circular piston ${ }^{\text {a) }}$
}

\author{
Takeru Yano and Yoshinori Inoue \\ Department of Engineering Science, Faculty of Engineering, Hokkaido University, Sapporo 060, Japan
}

(Received 13 April 1995; accepted for publication 30 January 1996)

\begin{abstract}
The propagation of nonlinear waves radiated by a circular piston mounted in an infinite plane rigid wall is numerically studied without the restriction of weak nonlinearity, in the case that the radius of piston is comparable with a typical wavelength of the radiated wave. The piston executes harmonic oscillations and the wave is thereby emitted into an ideal gas of semi-infinite extent, in which the dissipation effect is supposed to be negligible everywhere except for the discontinuous shock front. The wave phenomenon in the near field caused by the strongly nonlinear effect combined with the diffraction effect is clarified by solving the Euler equations with the upwind finite difference scheme. Owing to the strong nonlinearity, not only the waves emitted directly from the piston face but also the diffraction waves from the edge of the source are distorted and developed into the shock waves. This can lead to a multiple interference of shock waves in the near field. The separation phenomenon at the edge is also shown. Another remarkable phenomenon is the excitation of strong streaming (a mean mass flow), which forms a vortex-ring-like flow pattern and rarefies the gas near the source during the several periods of oscillation of the piston. By using a regular perturbation expansion, acoustic streaming in the weakly nonlinear problem is also analyzed, which does not show such a vortex-ring-like flow pattern and never rarefies the gas near the source.
\end{abstract} (C) 1996 Acoustical Society of America.

PACS numbers: 43.25.Vt, 43.25.Nm, 43.25.Cb, 43.25.Jh

\section{INTRODUCTION}

We shall consider the nonlinear propagation of sound waves radiated by a circular piston mounted in an infinite plane rigid wall. Most analyses for the nonlinear effect on such a diffractive wave radiated by a piston source have so far been confined to the weakly nonlinear and slightly diffractive acoustic beam, i.e., to the case that the wave amplitude is finite but sufficiently small and the source radius is sufficiently large compared with a typical wavelength. ${ }^{1-5}$ In this paper, we shall numerically investigate the propagation of the nonlinear waves emitted into an ideal gas from a harmonically oscillating circular piston without imposing the restriction of weak nonlinearity, in the case that the piston radius is comparable with a typical wavelength.

Precisely speaking, we shall consider the wave motion characterized by the conditions

$$
M \equiv \frac{a \omega}{c_{0}}=O(1), \quad \operatorname{Re} \equiv \frac{(\gamma+1) c_{0} a}{\delta^{*}} \gg 1,
$$

and

$$
R \equiv \frac{k R^{*}}{2 \pi}=O(1)
$$

where $M$ is the acoustic Mach number, Re is the acoustic Reynolds number, and $R$ is the ratio of the piston radius $R^{*}$ to a typical wavelength of the radiated sound $2 \pi / k=2 \pi c_{0} / \omega\left(c_{0}\right.$ is the speed of sound in an initial undis-

\footnotetext{
${ }^{a}$ A part of the work reported here is found in Advances in Nonlinear Acoustics, edited by H. Hobæk (World Scientific, Singapore, 1993), pp. 583588 .
}

turbed gas, $a$ and $\omega$ are an amplitude and an angular frequency of harmonic oscillation of the piston, $\gamma$ is the ratio of specific heats for the ideal gas, and $\delta^{*}$ is the diffusivity of sound $\left.{ }^{6}\right)$. The first condition $M=O(1)$ means that the wave is a strongly nonlinear one, i.e., its profile is rapidly distorted and this leads to the shock formation near the piston. The second condition Re $\gg 1$ means that we can regard the shock as a discontinuity, and may ignore the dissipation effect everywhere except for the discontinuity. ${ }^{7}$ The third condition $R=O(1)$ means that the diffraction effect cannot be neglected so that the wave cannot, even in the near field, ${ }^{8}$ be regarded as a collimated beam.

Recently, we have investigated the propagation of the strongly nonlinear plane wave of $M=O(1)$ radiated by an infinite plate oscillating harmonically, ${ }^{9}$ and the propagation of the strongly nonlinear spherical wave of $M=O(1)$ emitted from a harmonically pulsating sphere. ${ }^{10}$ In the present paper, we shall take up a piston problem. By applying a numerical method based on a high-resolution upwind finite difference scheme by Osher and Chakravarthy ${ }^{11,12}$ to the Euler equations (mass, momentum, and energy conservation laws), we shall clarify the wave phenomenon in the near field caused by the strongly nonlinear effect combined with the diffraction effect. We here remark that most of the relevant studies in the past have been focused on the far-field problems of $M \ll 1, \operatorname{Re} \gg 1$, and $R \gg 1$ (large transducer), where the parabolic approximation has often been utilized. ${ }^{1,3-5}$ In the present problem of $M=O(1), \mathrm{Re} \gg 1$, and $R=O(1)$, not only the waves emitted directly from the piston face but also the diffraction waves from the edge of the source are distorted and developed into the shock waves near the source. This leads to a complex phenomenon including a multiple 
interference (intersection) of shock waves in the near field (see Fig. 11 in Sec. III).

Furthermore, we examine acoustic streaming (a mean mass flow). We shall show that streaming forms a vortexring-like flow pattern in the near field, and that a timeaveraged density of the gas near the source is reduced by the streaming during the several periods of oscillation of the piston. Such a vortex-ring-like mass flow as is excited in the strongly nonlinear case does not appear in the steady state of the weakly nonlinear case of $M \ll 1$ and $\mathrm{Re} \gg 1$; this is verified by using a regular perturbation expansion in $M$ (see Appendices A-D). It is also shown that the gas near the source is not rarefied by the weakly nonlinear streaming. We emphasize here that the weakly nonlinear streaming occurs without any dissipation process, as streaming analyzed in Refs. 1316. The maximum value (maximum velocity) of streaming in the strongly nonlinear case is considerably large compared with that of streaming caused by the strongly nonlinear plane waves with the same $M{ }^{9}$

In Ref. 17, Lighthill pointed out that the strong streaming motion in the far field generated by a powerful narrow sound beam becomes a turbulent jet. Although we don't examine the far-field behavior of streaming, the turbulent streaming jet may not be formed in the far field of the present problem, because the wave motion concerned is highly diffractive $[R=O(1)]$ and hence the wave in the far field may rather be a spherically diverging one than a narrow beam. Another important remark was made in Ref. 17: the strong streaming motion ${ }^{18}$ should be analyzed without neglecting the inertia term (nonlinear term) in the governing equations of streaming, which are the equations of viscous incompressible flow with the driving force being determined from the sound field. ${ }^{17}$ In the present paper, we shall not use the equations of streaming but numerically solve the fundamental equations of gas dynamics, i.e., the Euler equations. This procedure is allowed by the condition $\operatorname{Re} \gg 1$.

We here comment on a difficulty inherent in the problem of a circular piston of planar face mounted in a plane rigid wall; the difficulty does not seem to have been discussed. The edge of the source forms a convex right-angled corner in the $\left(x^{*}, r^{*}\right)$ plane (see Fig. 1). In inviscid potential flows, the fluid velocity becomes infinity at such a corner, ${ }^{19}$ and in high Reynolds number viscous flows the vertex of the corner is a separation point of boundary layer. ${ }^{20} \mathrm{We}$ shall show that, in the linear problem of Re $\gg 1$ (potential flow), the radial component of the fluid velocity logarithmically diverges at the edge (see Sec. II and Appendix B). In the analytical treatment for the linear case and the weakly nonlinear case of $M \ll 1$ and $\operatorname{Re} \gg 1$, instead of eliminating the singularity, we can confirm that the obtained result is correct except for the small neighborhood of the singular point (see Appendices A-D). The numerical results for the strongly nonlinear case of $M=O(1)$ and $\operatorname{Re} \gg 1$ show the separation phenomenon and formation of vortex. Although the viscous effect is not included in the Euler equations, solutions containing separation at sharp trailing edge are obtained in many computational researches, ${ }^{21,22}$ without invoking a Kutta condition, ${ }^{23}$ which states that for high Reynolds number flow past a sharp trailing edge the flow leaves the body at the edge indepen-

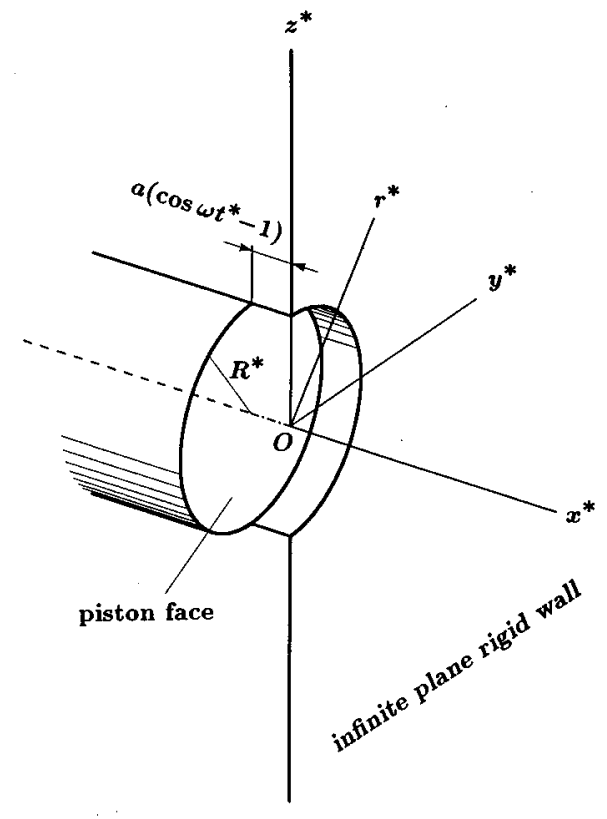

FIG. 1. Cutaway view: circular piston with a planar face of radius $R^{*}$ is mounted in an infinite plane rigid wall. The piston executes a harmonic oscillation such that the displacement of its face from the wall at time $t^{*}$ is $a\left(\cos \omega t^{*}-1\right)$

dent of the Reynolds number and the edge can be a source of vorticity. The Euler equations admit vortex sheets as weak solutions, so that vortex sheets can be "captured" in the same sense that shocks can be captured and a Kutta condition need not be enforced explicitly. In the solution of the Euler equations, the separation is signified by the vortex sheet being shed from the edge. ${ }^{24}$ The numerical result shown in Sec. III C seems to be correct at least qualitatively, although the vortex sheet is smeared out over mesh cells by the numerical dissipation.

In Sec. I, the problem is formulated mathematically. In Sec. II, the familiar results for the linear wave are recalled for comparison with the strongly nonlinear wave examined in Sec. III. The flow pattern for weakly nonlinear streaming is also shown. In Sec. III, we shall present the results of the numerical computation for the strongly nonlinear waves. The profiles are contrasted with those of the linear solution, and the on-axis profiles are compared with the profiles of the strongly nonlinear plane wave. The evolution and interference of shock waves are demonstrated. The excitation of strong streaming and production of vorticity at the shock front are also analyzed in this section. The numerical solution displays the separation phenomenon at the edge. In Sec. IV, we shall summarize the main results. The reformulation of the problem for the weakly nonlinear waves and an analytical expression for weakly nonlinear acoustic streaming are presented in Appendices A-D. The numerical method is briefly explained in Appendix E.

\section{FORMULATION OF THE PROBLEM}

In order to formulate the problem, we shall introduce the following nondimensional variables: 

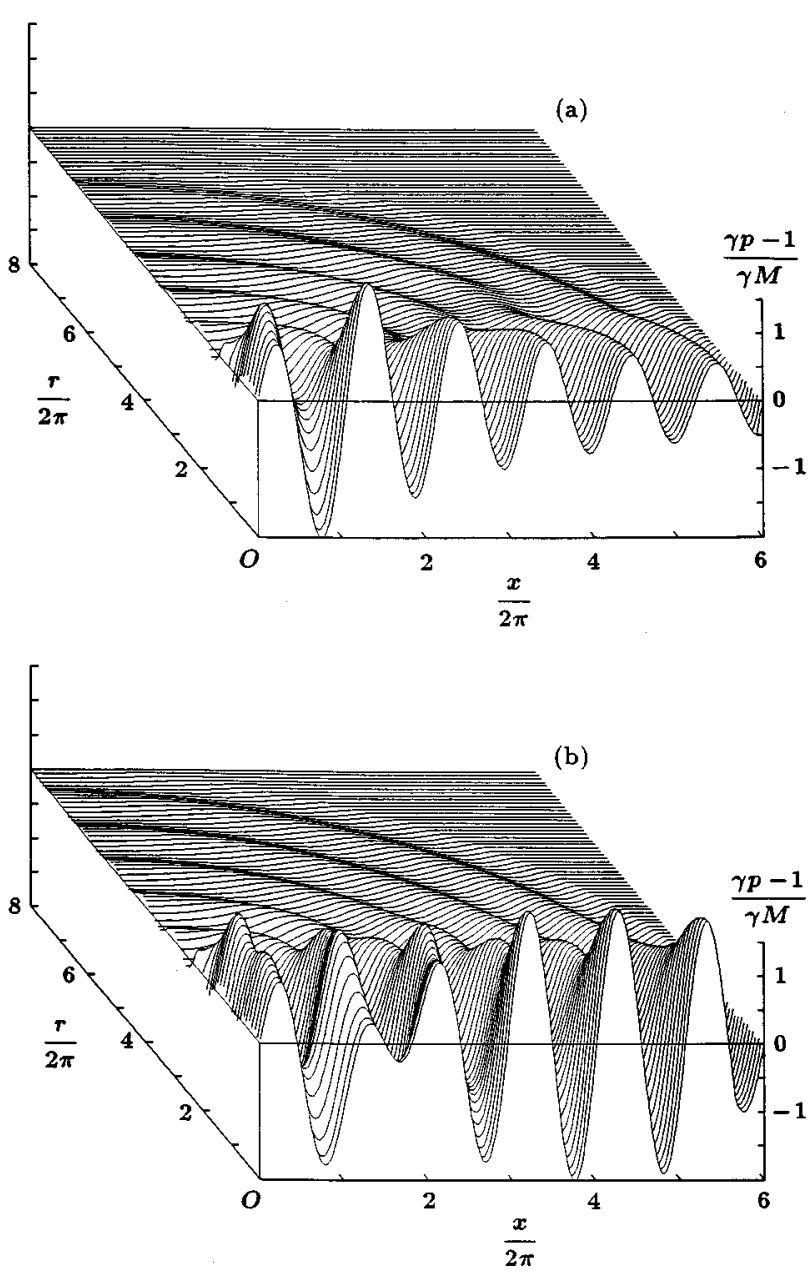

$$
\begin{aligned}
& t=\omega t^{*}, \quad x=\frac{x^{*} \omega}{c_{0}}, \quad r=\frac{r^{*} \omega}{c_{0}}, \quad u=\frac{u^{*}}{c_{0}}, \\
& v=\frac{v^{*}}{c_{0}}, \quad \rho=\frac{\rho^{*}}{\rho_{0}}, \quad p=\frac{p^{*}}{\rho_{0} c_{0}^{2}},
\end{aligned}
$$

where $x^{*}$ is the distance from the wall, $r^{*}$ is the distance from the axis of the piston, i.e., the $x^{*}$ axis (see Fig. 1), $u^{*}$ and $v^{*}$ are, respectively, the $x^{*}$ and $r^{*}$ components of the fluid velocity, $\rho^{*}$ is the density of the gas, and $p^{*}$ is the pressure ( $\rho_{0}$ is an initial undisturbed density).

The condition $\mathrm{Re} \gg 1$ allows neglecting the energy dissipation effect due to viscosity and thermal conductivity of the gas until a shock wave emerges in the wave field. Hence, at least until the time of shock formation, we can use the system of Euler equations for the flow with cylindrical symmetry around the $x$ axis:

$$
\begin{aligned}
& \frac{\partial \rho}{\partial t}+\frac{\partial(\rho u)}{\partial x}+\frac{\partial(\rho v)}{\partial r}+\frac{\rho v}{r}=0, \\
& \frac{\partial(\rho u)}{\partial t}+\frac{\partial\left(p+\rho u^{2}\right)}{\partial x}+\frac{\partial(\rho u v)}{\partial r}+\frac{\rho u v}{r}=0, \\
& \frac{\partial(\rho v)}{\partial t}+\frac{\partial(\rho u v)}{\partial x}+\frac{\partial\left(p+\rho v^{2}\right)}{\partial r}+\frac{\rho v^{2}}{r}=0,
\end{aligned}
$$

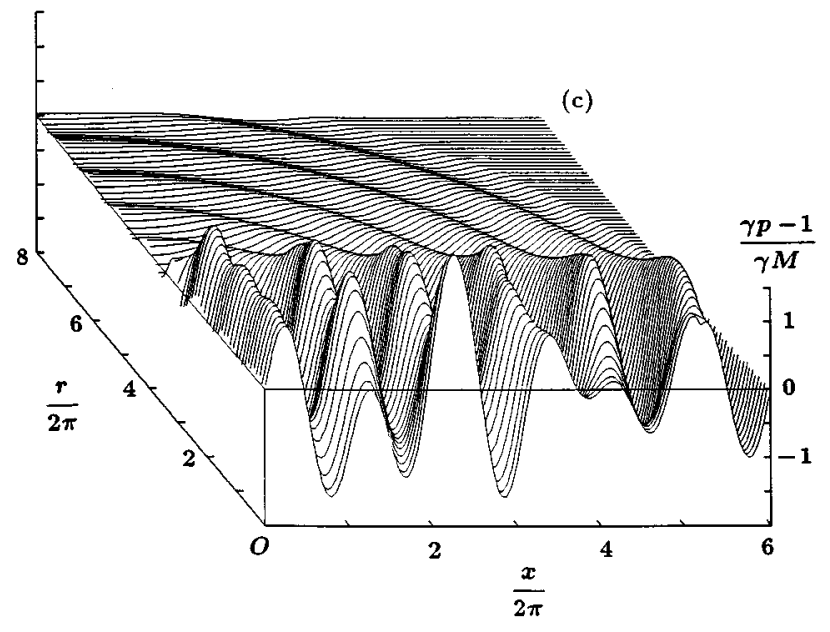

FIG. 2. Profile of the pressure at $t=12 \pi$ in the linear case of $M \rightarrow 0$ : (a) $R=1$, (b) $R=2$, and (c) $R=3$.

$$
\frac{\partial E_{t}}{\partial t}+\frac{\partial\left[\left(E_{t}+p\right) u\right]}{\partial x}+\frac{\partial\left[\left(E_{t}+p\right) v\right]}{\partial r}+\frac{\left(E_{t}+p\right) v}{r}=0,
$$

where $E_{t}=\rho\left(u^{2}+v^{2}\right) / 2+p /(\gamma-1)$ is the normalized total energy of the ideal gas per unit volume. Once a shock is formed, the energy dissipation can no longer be ignored,

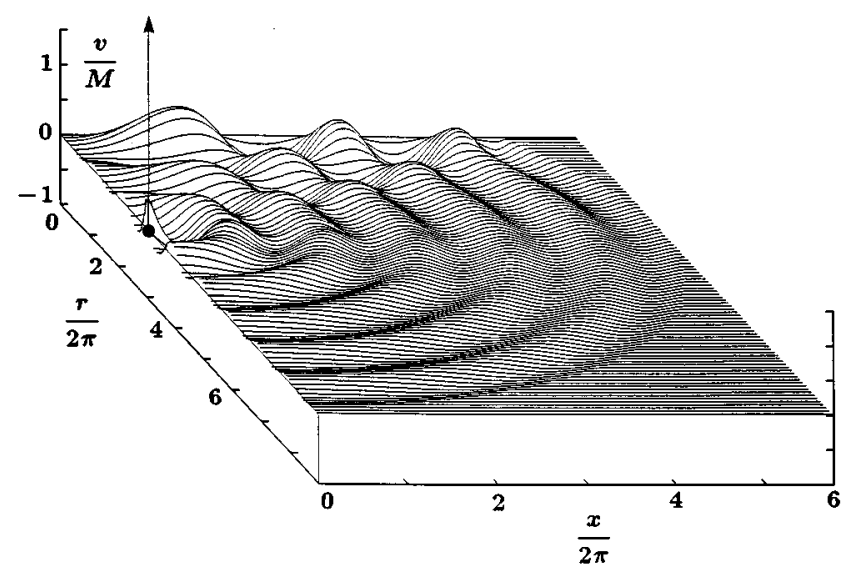

FIG. 3. Radial component of the fluid velocity for $R=3$ at $t=11.5 \pi$ in the linear case of $M \rightarrow 0$. A small closed circle denotes the singular point $(x, r)$ $=(0,2 \pi R)$. 

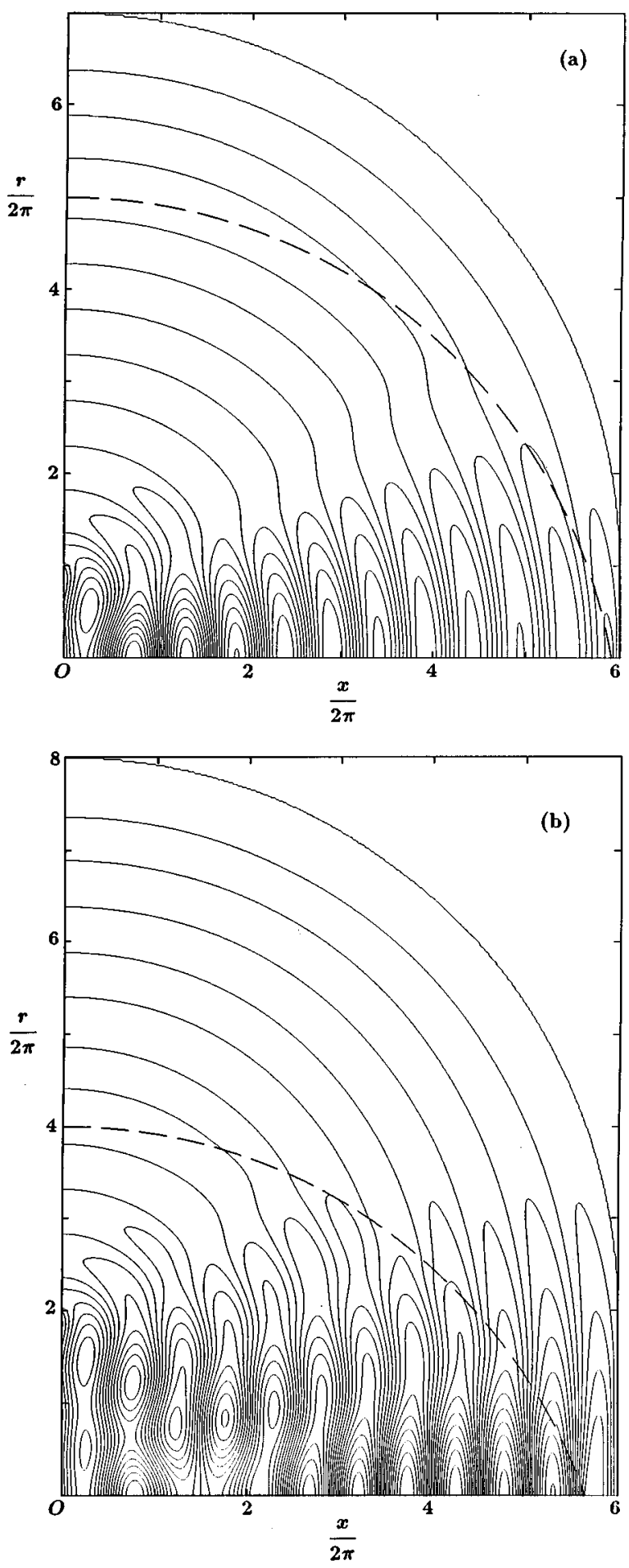

which must in reality be produced at least at the shock front. As is generally known, however, a shock solution can be represented as a discontinuity in the system of equations (3)(6), owing to the condition $\mathrm{Re} \gg 1$. We can thus employ this system even after the shock formation time. We shall numerically obtain such a discontinuous solution by using the high-resolution upwind finite difference scheme, ${ }^{11,12}$ under the initial and boundary conditions described below.

The gas is supposed to be uniform and at rest for $t \leqslant 0$. The initial conditions at $t=0$ are therefore

$$
u=v=0, \quad \rho=1, \quad p=1 / \gamma \quad(x \geqslant 0) .
$$

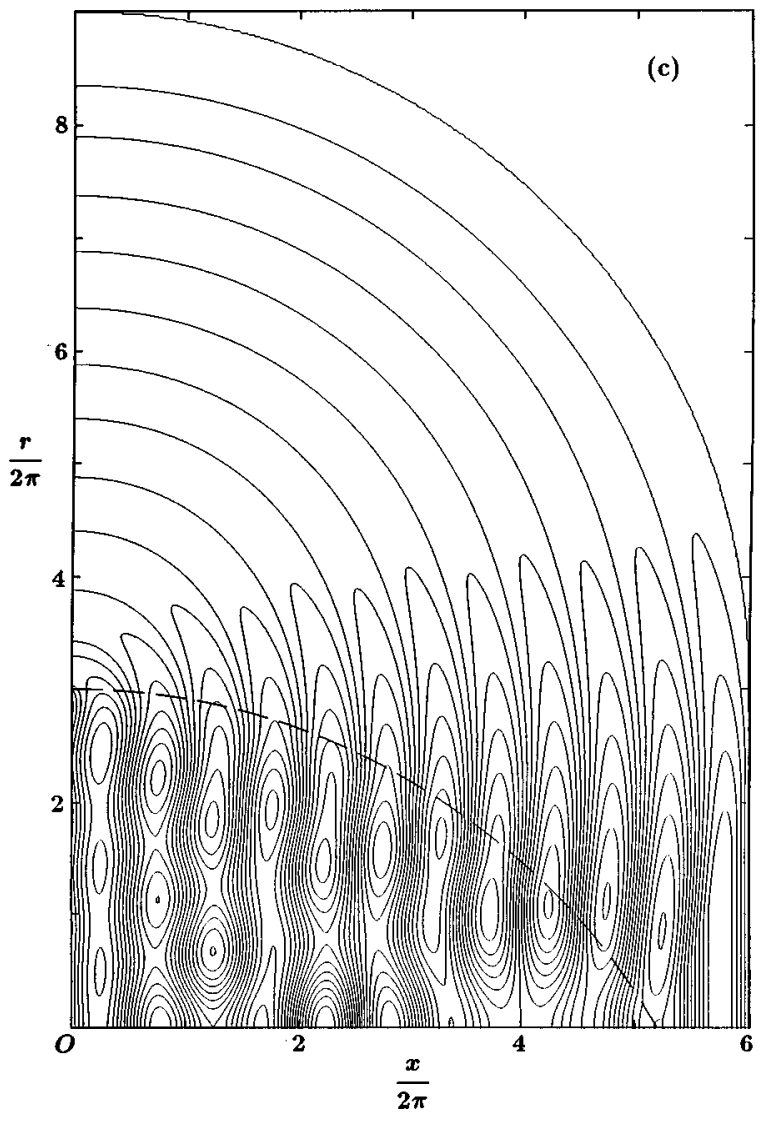

FIG. 4. Pressure contour at $t=12 \pi$ in the linear case of $M \rightarrow 0$. The normalized pressure $p_{1}$ is plotted in steps of 0.2 . The dashed curve is the boundary between the steady and transient state: (a) $R=1$, (b) $R=2$, and (c) $R=3$.

We shall take up the case that the planar piston is mounted in the plane rigid wall. The instantaneous location of the piston face, $X(t)$, is given by

$$
X(t)=M(\cos t-1) \quad(t>0)
$$

(see Fig. 1). The boundary conditions on the piston surface and on the wall may therefore be expressed as follows:

$$
\begin{aligned}
& u=-H(2 \pi R-r) H(r) M \sin t \\
& \quad \text { at } \quad x=H(2 \pi R-r) H(r) X(t) \quad(t>0),
\end{aligned}
$$


$\rightarrow M^{2}$

(a)

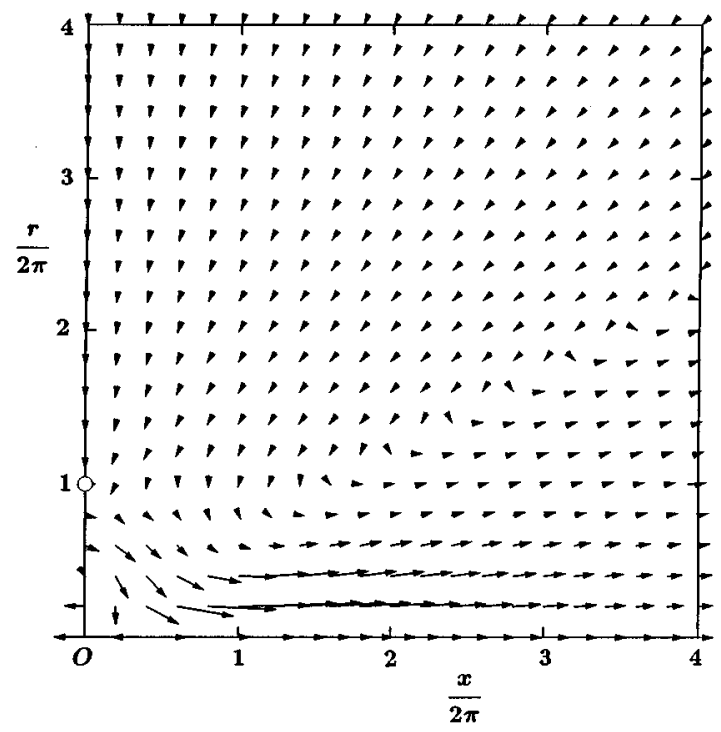

$\rightarrow$

(b)

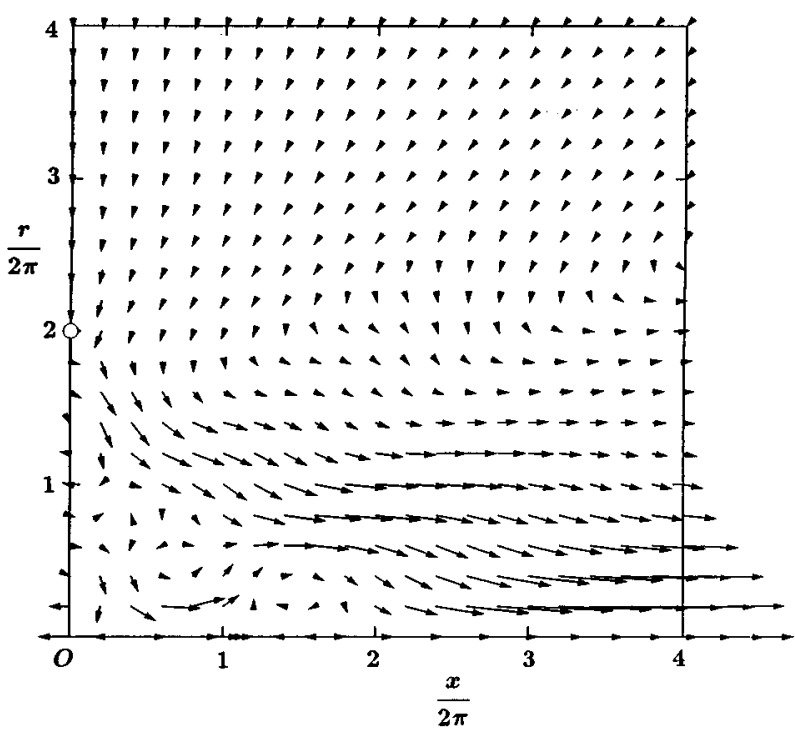

$v=0 \quad$ at $\quad r=2 \pi R \quad$ for $\quad X(t)<x<0 \quad(t>0)$,

where $H(r)$ is the Heaviside unit step function defined as

$$
H_{r}= \begin{cases}1 & (r>0), \\ 0 & (r<0) .\end{cases}
$$

In addition, owing to the continuity and axial symmetry, we let $v=0$ on the $x$ axis.

\section{WEAKLY NONLINEAR PROBLEM IN THE NEAR FIELD}

Before proceeding to the strongly nonlinear waves, we shall discuss some essential properties of the weakly nonlinear wave of $M \ll 1, \operatorname{Re} \gg 1$, and $R=O(1)$ in the near field. For the case of the weakly nonlinear waves, we reformulate the problem in terms of the velocity potential (see Appendix A).

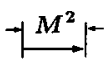

(c)

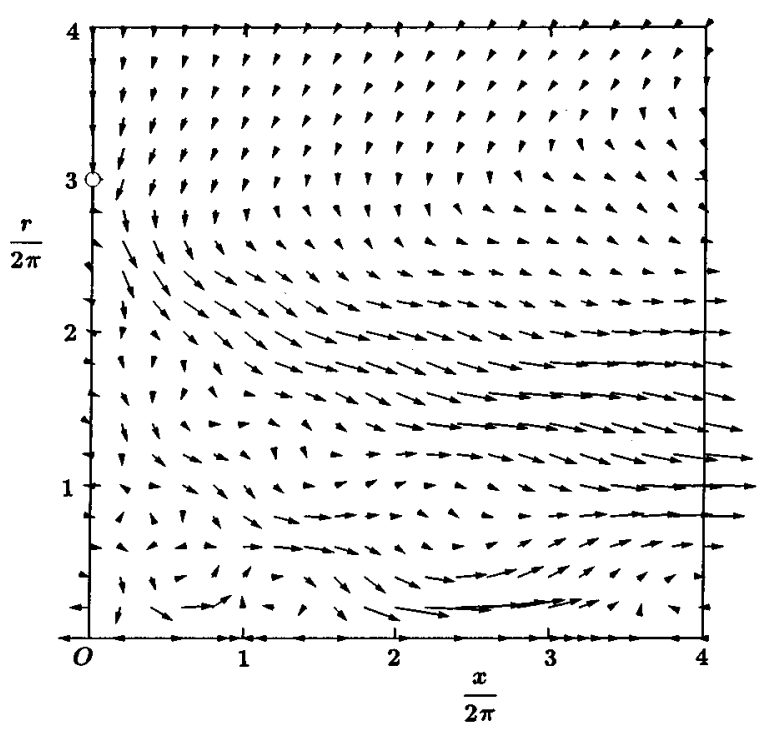

FIG. 5. Weakly nonlinear acoustic streaming. Arrows signify the timeaveraged mass flux density vector $\mathbf{v}_{s}$ to the approximation of $O\left(M^{2}\right)$. The singular point, $(x, r)=(0,2 \pi R)$, is marked with a small open circle. A reference arrow, the size of which corresponds to $\left|\mathbf{v}_{s} / M^{2}\right|=1$, is shown on the top of each figure: (a) $R=1$, (b) $R=2$, and (c) $R=3$.
Applying a regular perturbation expansion in $M$, we can obtain analytical results to a certain extent for the near field (Appendices B-D).

\section{A. Linear propagation in the near field}

In the near field of the weakly nonlinear waves, the distortion of wave profile due to the nonlinear effect may be neglected, i.e., the propagation process is linear at least to the leading order of approximation. We therefore take up the linear propagation, which has been well examined by many authors, and the propagation property of which is now familiar (for the review of representative contributions, see Refs. 25 and 26). One of the most important phenomena in the near field of the linear wave is the diffraction and the interference resulting from it, which we demonstrate here for comparison with the strongly nonlinear waves examined in Sec. III. 

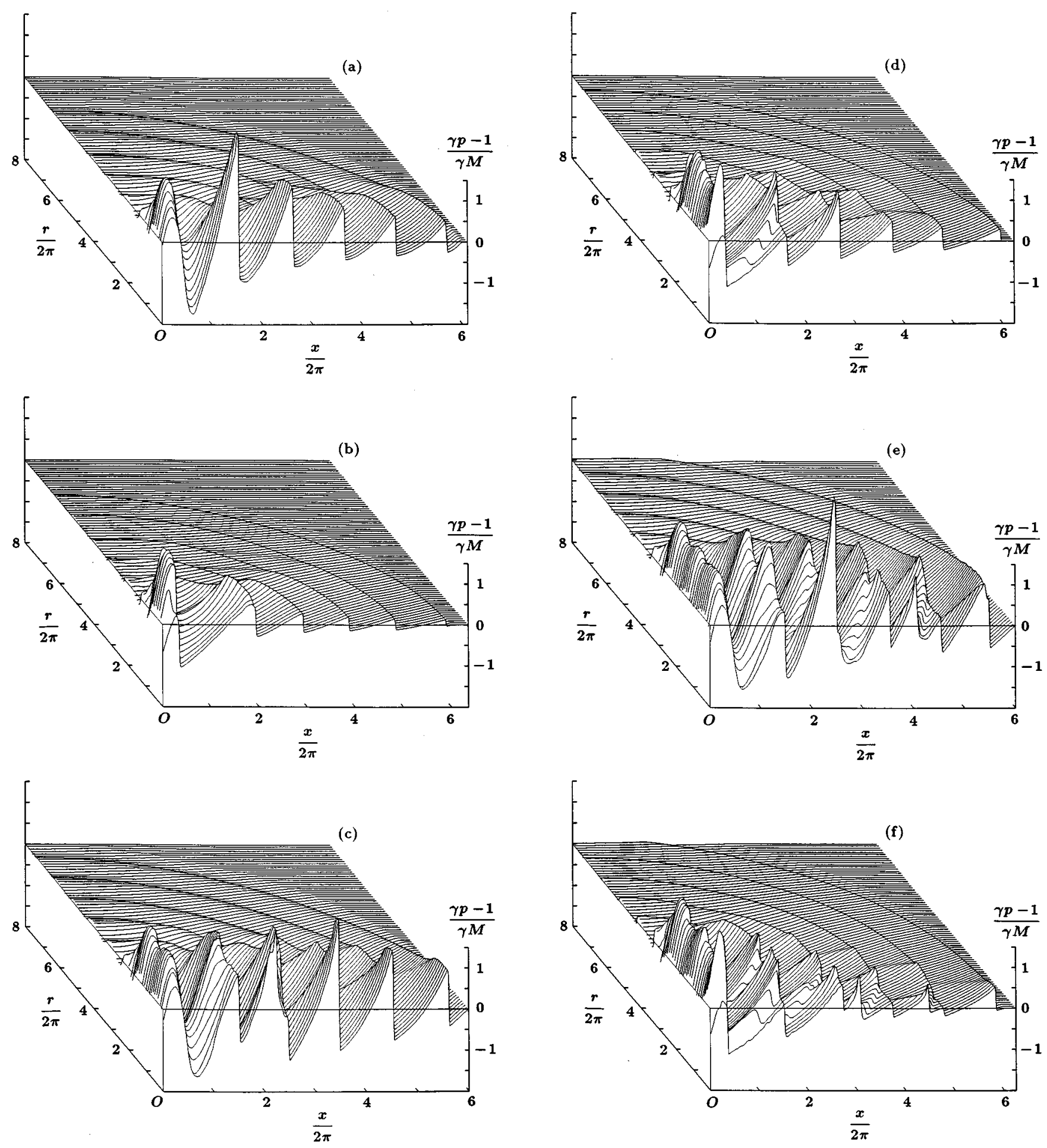

FIG. 6. Pressure profile at $t=12 \pi$ in the strongly nonlinear case: (a) $R=1$ and $M=0.1$, (b) $R=1$ and $M=0.4$, (c) $R=2$ and $M=0.1$, (d) $R=2$ and $M=0.4$, (e) $R=3$ and $M=0.1$, and (f) $R=3$ and $M=0.4$.

In Appendix B, the linear solution is expressed in the form of summation of a plane wave and a wave originating from the edge of the source (a diffraction wave). The linear wave forms shown in Figs. 2-4 are obtained by numerical integrations for Eqs. (B11), (B13), (B14), and (B16) in Appendix B.

The pressure profile of the linear wave at $t=12 \pi$ is shown in Fig. 2, where a normalized sound pressure $(\gamma p-1) / \gamma M=p_{1}$ (in the limit as $M \rightarrow 0$ ) given by Eqs.
(B11) and (B14) in Appendix B is plotted (the subscript 1 signifies the first-order term in a regular perturbation expansion in $M$, see Appendix A). With increase in $R$, the complexity of the wave field due to interference increases in a region $0<x$ and $0 \leqslant r \leqslant 2 \pi R$ as demonstrated in Refs. 25 and 27.

In Fig. 3, we depict the profile of the radial component of the fluid velocity $v / M=v_{1}$ (in the limit as $M \rightarrow 0$ ) at $t=11.5 \pi$, which is given by Eqs. (B13) and (B16) in Appen- 


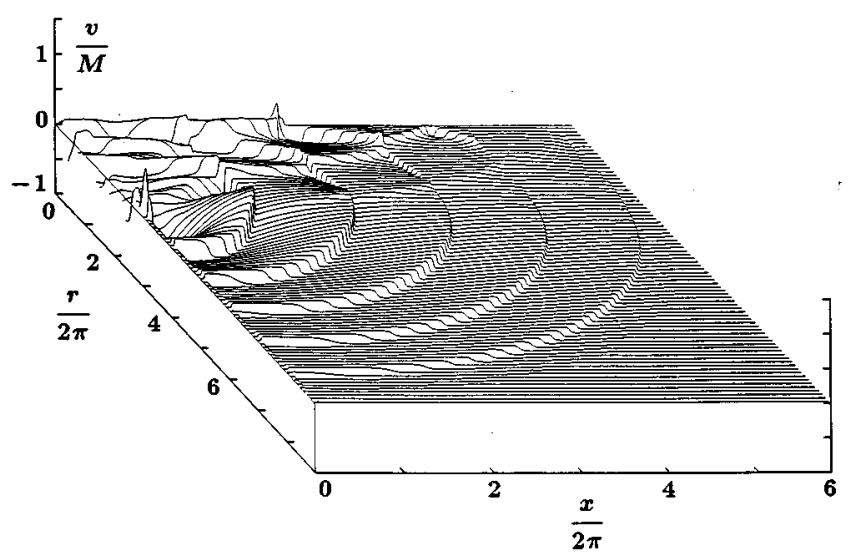

FIG. 7. Radial velocity at $t=11.5 \pi$ in the strongly nonlinear case of $R=3$ and $M=0.4$.
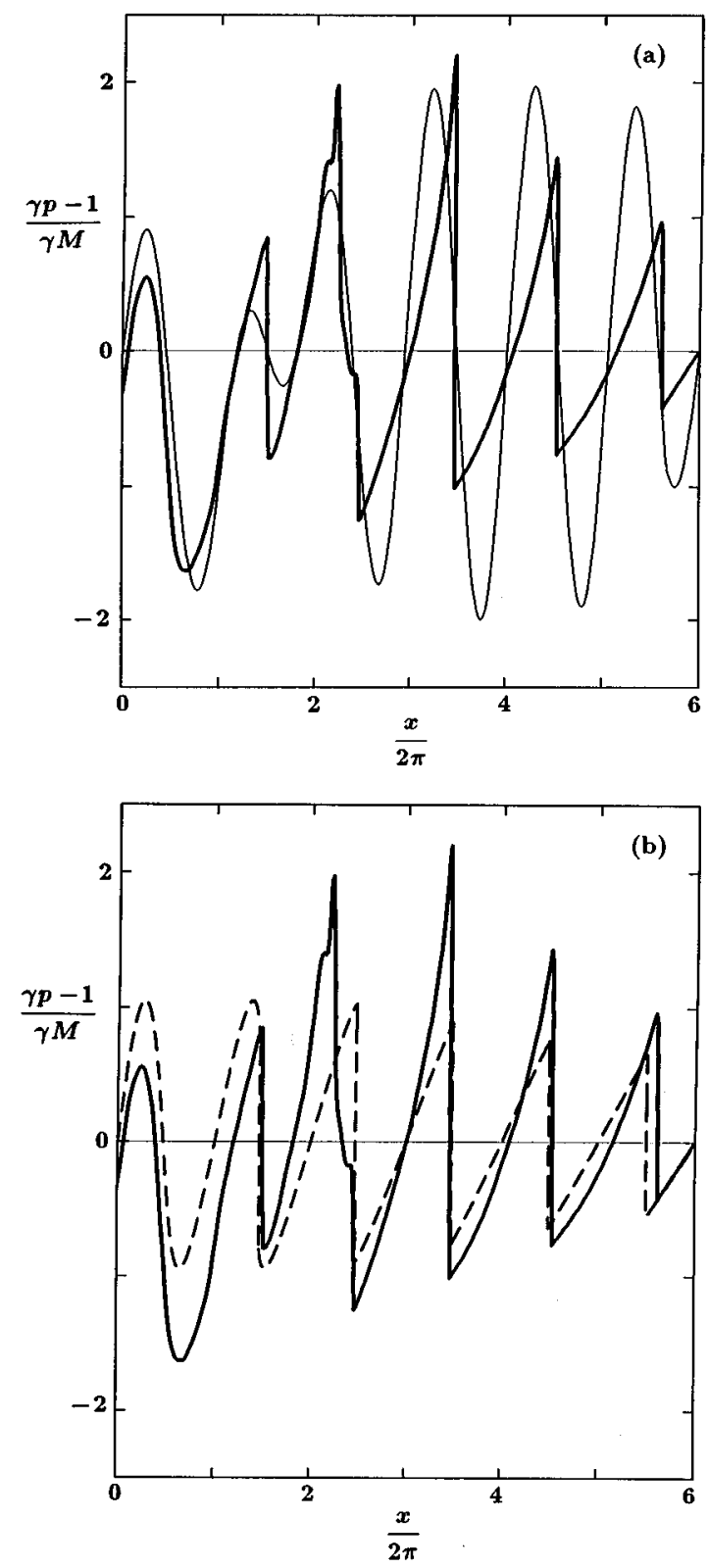

FIG. 8. Pressure profile on the axis at $t=12 \pi$. The strongly nonlinear wave of $R=2$ and $M=0.1$ denoted by a bold solid curve is compared with (a) the linear wave of $R=2$ denoted by a thin solid curve, and with (b) the strongly nonlinear plane wave of $M=0.1$ denoted by a dashed curve (cf. Ref. 9).
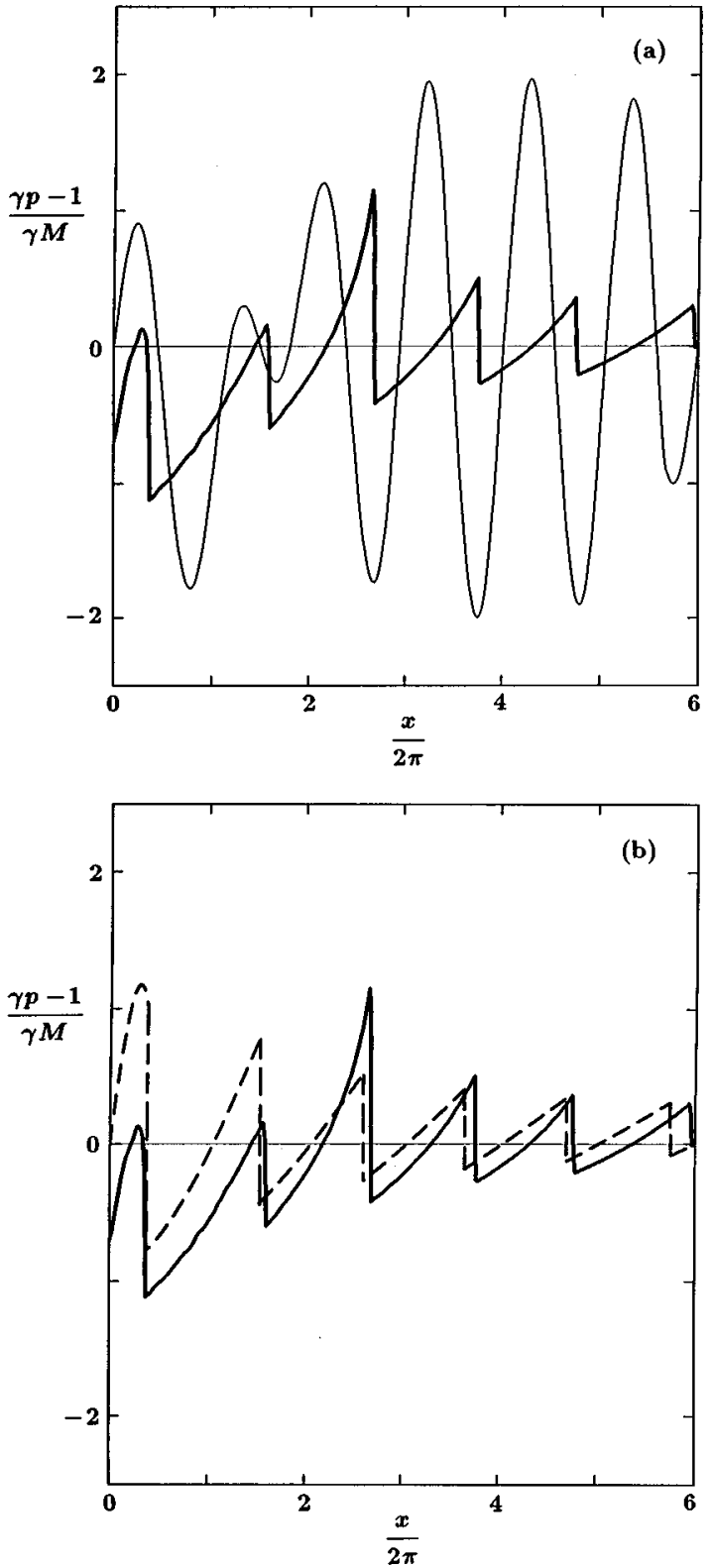

FIG. 9. Pressure profile on the axis at $t=12 \pi$. The strongly nonlinear wave of $R=2$ and $M=0.4$ denoted by a bold solid curve is compared with (a) the linear wave of $R=2$ denoted by a thin solid curve, and with (b) the strongly nonlinear plane wave of $M=0.4$ denoted by a dashed curve (cf. Ref. 9).

dix B. As shown in Appendix B, $v_{1}$ diverges logarithmically at $(x, r)=(0,2 \pi R)$, so that $v_{1}$ is not plotted at the point in Fig. 3. However, as can be seen from Fig. 3, $v_{1}$ remains of the order of unity everywhere except for a neighborhood of the singular point, because the rate of divergence is very slow. Owing to this fact, we may regard the solution as the correct one except for the very small neighborhood of the singular point.

Figure 4 shows the contour of the pressure $p_{1}$ at $t=12 \pi$, plotted in steps of 0.2. The dashed curve denotes the boundary between a steady-state region and a transient region at $t=12 \pi$, i.e., $p_{1}(x, r, t)=p_{1}(x, r, t+2 \pi)$ for $t \geqslant 12 \pi$ in the region enclosed with the dashed curve (Appendix B). As $R$ increases, the complicated interference pattern is formed within a region $0<x$ and $0 \leqslant r \leqslant 2 \pi R$. The diffraction wave 

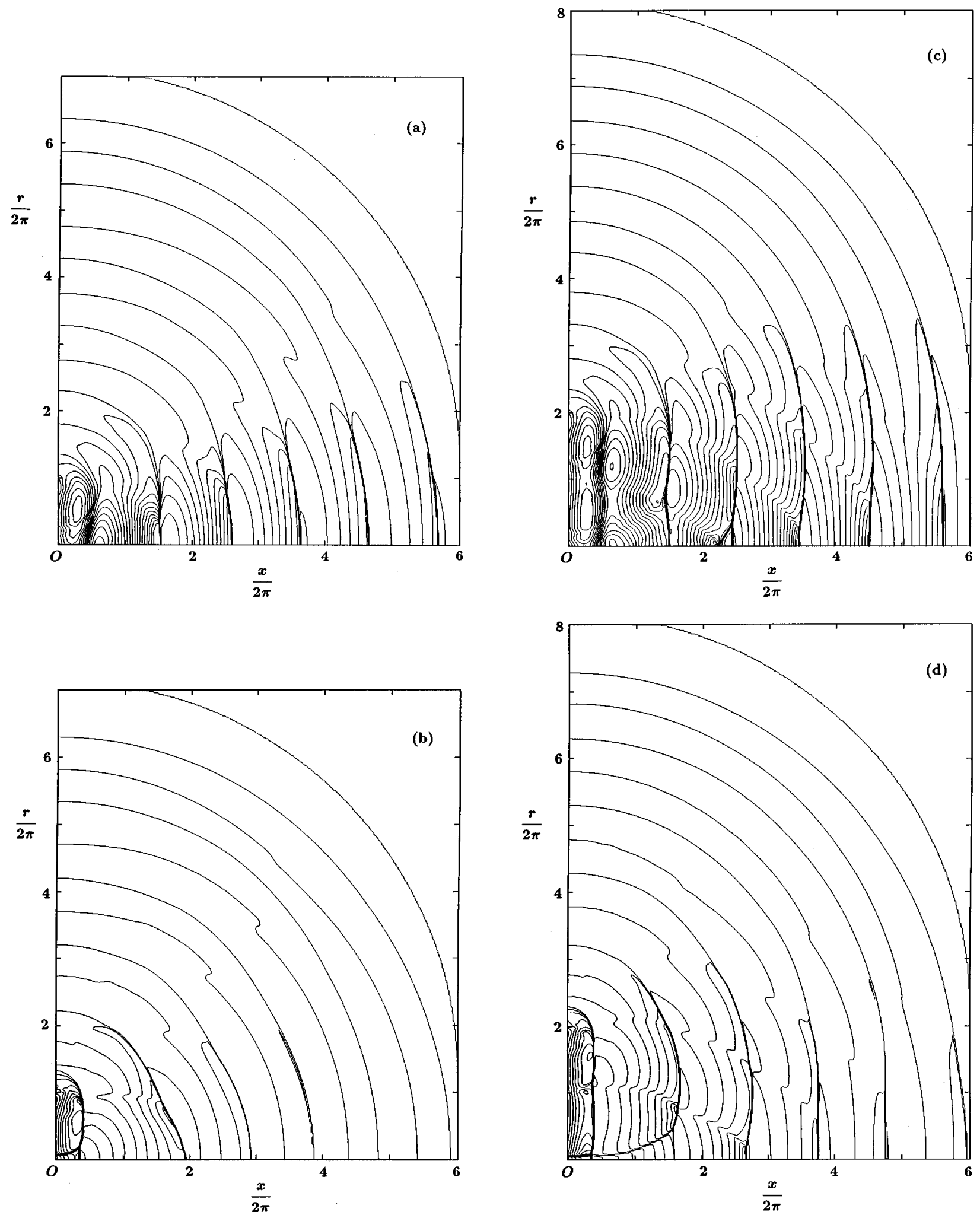

FIG. 10. Pressure contour in the strongly nonlinear case. The normalized pressure $(\gamma p-1) / \gamma M$ is plotted in steps of 0.2 : (a) $R=1$ and $M=0.1$, (b) $R=1$ and $M=0.4$, (c) $R=2$ and $M=0.1$, (d) $R=2$ and $M=0.4$, (e) $R=3$ and $M=0.1$, and (f) $R=3$ and $M=0.4$. 

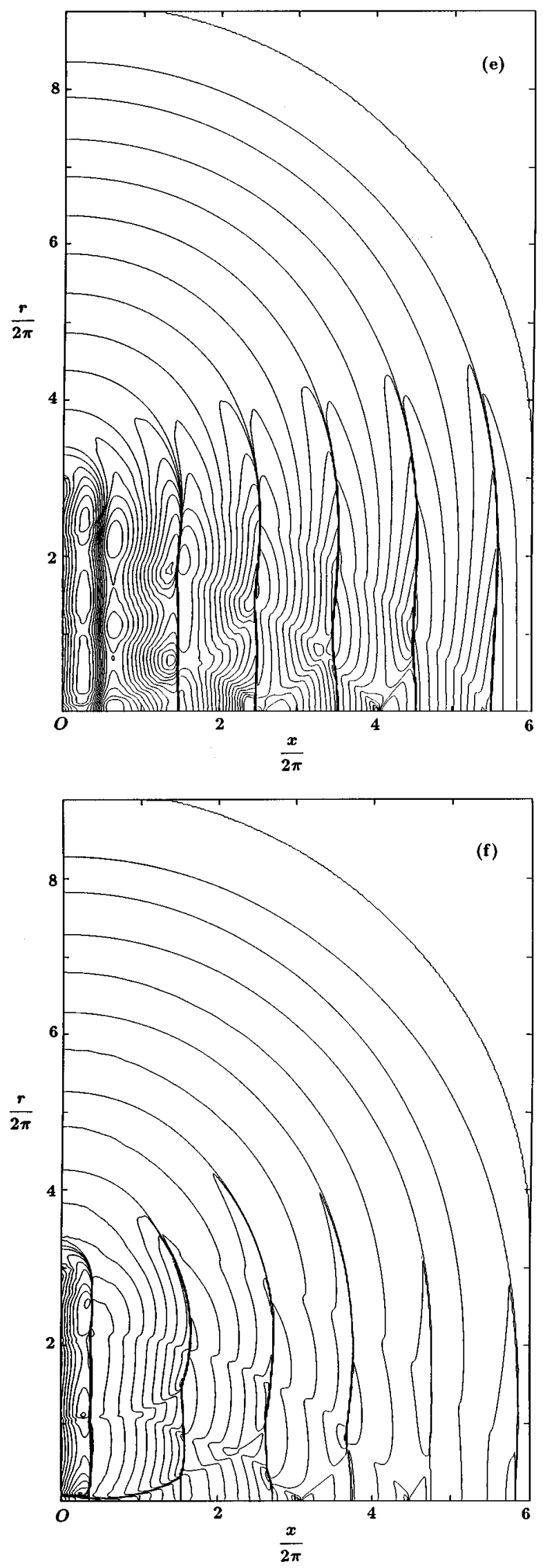

FIG. 10. (Continued.) outside the region looks like a spherical wave emitted from a source located at $(x, r)=(0,2 \pi R)$.

\section{B. Weakly nonlinear acoustic streaming}

We shall examine weakly nonlinear acoustic streaming, in line with the method used in Refs. 13-16. To do so, we shall define a time-averaged mass flux density vector $\mathbf{v}_{s}$ $\equiv(\overline{\rho u}, \overline{\rho v})$ as

$$
\begin{aligned}
& \overline{\rho u}(x, r, t ; T)=\frac{1}{T} \int_{t-T}^{t} \rho(x, r, \tau) u(x, r, \tau) d \tau, \\
& \overline{\rho v}(x, r, t ; T)=\frac{1}{T} \int_{t-T}^{t} \rho(x, r, \tau) v(x, r, \tau) d \tau,
\end{aligned}
$$

where $T$ is an interval for time average.

For $t>\sqrt{x^{2}+(2 \pi R+r)^{2}}$ for a given point $(x, r)$, the linear wave motion becomes periodical with period $2 \pi / \omega$ (Appendix B). In what follows, we assume that $t$ is sufficiently large, and we let $T=2 \pi$. To the approximation of $O\left(M^{2}\right)$, Eq. (12) can then be reduced to

$$
\mathbf{v}_{s}=M^{2}\left(\overline{\rho_{1} u_{1}}+\overline{u_{2}}, \overline{\rho_{1} u_{1}}+\overline{v_{2}}\right),
$$

where the bar denotes the mean value in the sense of Eq. (12), and the subscript 2 signifies the second-order term in a regular perturbation expansion in $M$ (see Appendix A). Consequently, $\mathbf{v}_{s}$ is independent of $t$ to the approximation of $O\left(M^{2}\right)$. In the strongly nonlinear case, however, $\mathbf{v}_{s}$ weakly depends on both $t$ and $T$ (see Sec. III B).

The explicit representations for $\bar{u}_{2}$ and $\bar{v}_{2}$ are given by Eqs. (C8) and (C9) in Appendix C, and those for $\overline{\rho_{1} u_{1}}$ and $\overline{\rho_{1} v_{1}}$ are given by Eqs. (D1)-(D4) in Appendix D. The flow patterns are shown by arrows in Fig. 5, which are obtained by numerical integrations. We have numerically confirmed that the mean mass flow $\mathbf{v}_{s}$ is divergence-free vector field (as incompressible flow), and that the total net mass flow across the mean surface of the piston face is zero (see Appendix C). Clearly, such a "steady" flow cannot rarefy the gas in the field.

Figure 5 shows that the mean mass flow $\mathbf{v}_{s}$ meanders and locally flows backward, which forms a contrast to the well-known Eckart streaming ${ }^{28}$ (note that the large scale vortexes in Eckart streaming are ascribed to the viscosity and the fact that the medium is contained in a cylinder of finite length). With increase in $R$, such a fine "structure" of the flow pattern becomes conspicuous in front of the piston face [Fig. 5(b) and (c)]. We shall emphasize that the weakly nonlinear streaming occurs without any dissipation process, contrary to Eckart streaming (see also Refs. 13-16).

\section{STRONGLY NONLINEAR WAVES AND STRONG STREAMING}

In this section, we shall present some typical numerical results for the strongly nonlinear waves of $M=O(1), \mathrm{Re} \gg 1$, and $R=O(1)$. The numerical method used here is based on a high-resolution upwind finite difference scheme by Osher and Chakravarthy, ${ }^{11,12}$ which has recently been applied by the present authors to the problems of the strongly nonlinear plane waves ${ }^{9}$ and spherical waves. ${ }^{10}$ The computations were 
(a)

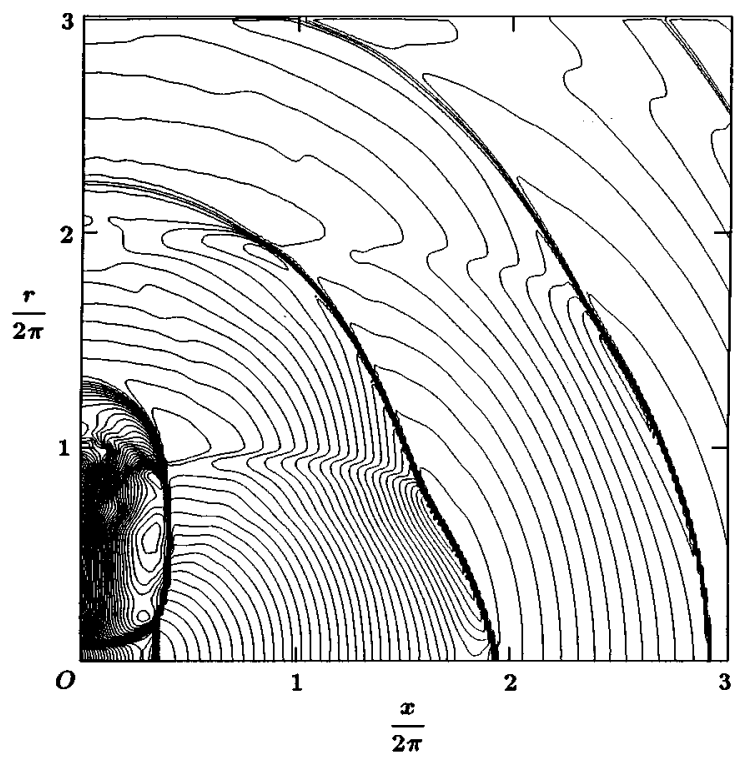

(b)

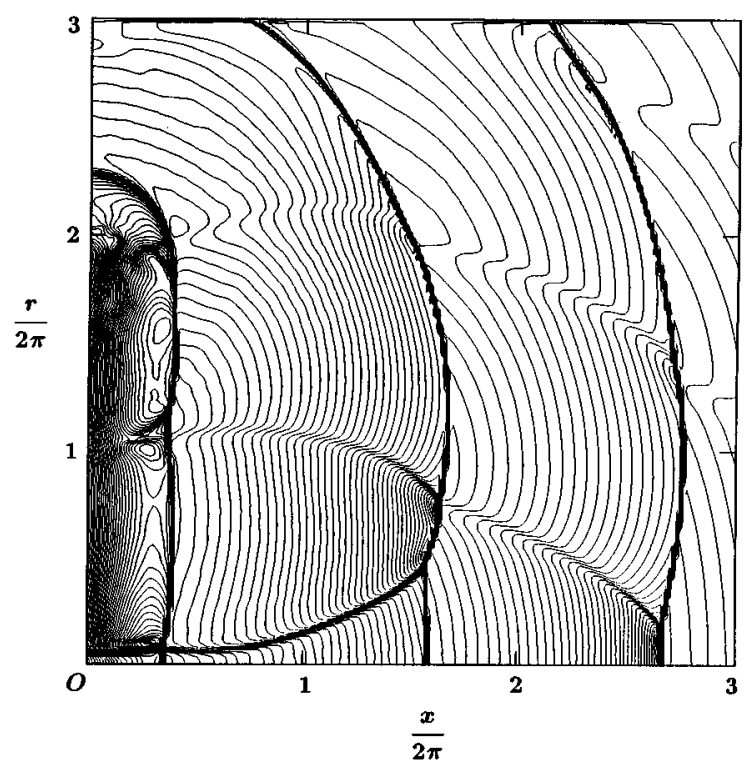

performed on the supercomputer HITAC S-820 at Hokkaido University. The numerical implementation is briefly shown in Appendix E. The ratio of specific heats $\gamma$ is fixed at $\gamma=1.4$ (air) for all computations.

\section{A. Evolution and interference of shock waves}

The pressure profiles at $t=12 \pi$ are shown in Fig. 6, in which one can see that, as in the linear case, the strongly nonlinear wave field can also be regarded as the summation of the wave directly radiated from the piston and the diffraction wave, i.e., the wave emitted from the edge of the piston (see also Fig. 10). Owing to the strongly nonlinear effect, the wave directly radiated from the piston is immediately distorted and develops into the shock in the near field. For $M=0.1$, an anomalous positive pressure peak emerges on the axis near the piston [Fig. 6(a), (c), and (e)]. As $M$ increases, the shock becomes strong and hence the energy dissipation at the shock front also increases, which results in the consider- (c)

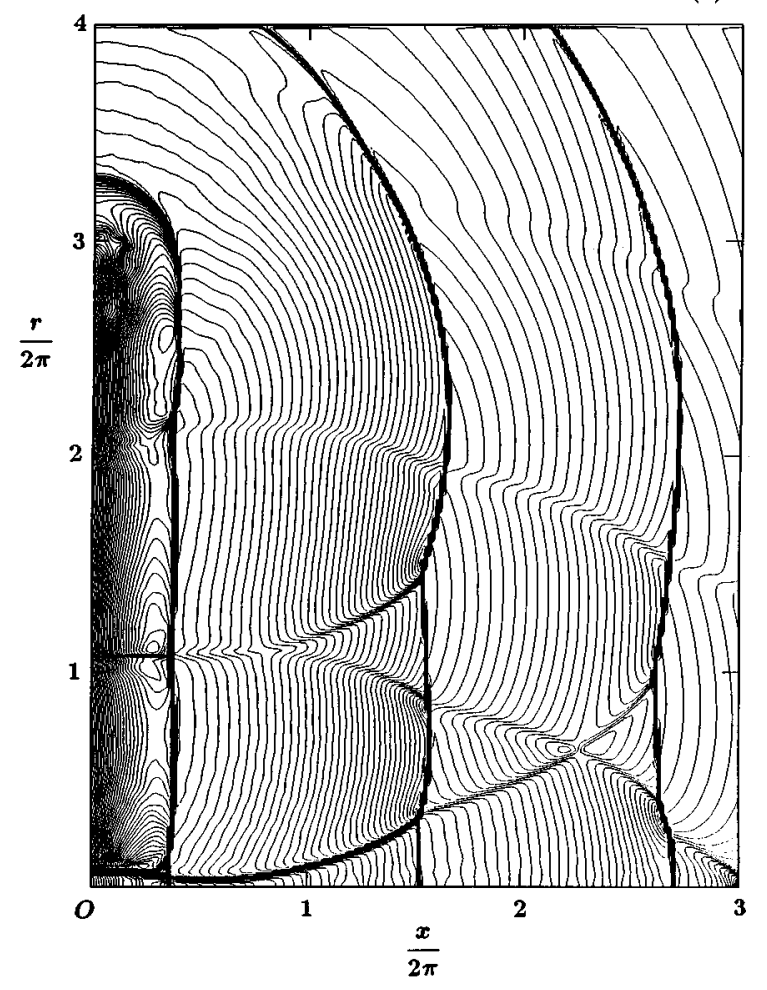

FIG. 11. The magnification of Fig. 10, emphasizing the intersection of shocks in the vicinity of the source: (a) $R=1$ and $M=0.4$, (b) $R=2$ and $M=0.4$, and (c) $R=3$ and $M=0.4$.

able decay of the wave amplitude [Fig. 6(b), (d), and (f)]. For $R \geqslant 2$, the strong nonlinearity appreciably distorts the diffraction waves [see Fig. 6(c)-(f)]. When $M$ increases to 0.4, the shock front has a rugged crest with spiky bump [Fig. 6(d) and (f)], and the profile is entirely different from the linear one (cf. Fig. 2). It can be seen from Fig. 6(e) and (f) that, on

TABLE I. The maximum value of the time-averaged mass flux density.

\begin{tabular}{ccccc}
\hline \hline Acoustic & \multicolumn{3}{c}{ Present result } & \\
\cline { 2 - 4 } $\begin{array}{c}\text { Mach number } \\
M\end{array}$ & $R=1$ & $R=2$ & $R=3$ & $\begin{array}{c}\text { Strongly nonlinear } \\
\text { plane wave }\end{array}$ \\
\cline { 2 - 4 } 0.1 & 0.037 & 0.018 & 0.018 & 0.002 \\
0.2 & 0.207 & 0.067 & 0.113 & 0.009 \\
0.3 & 0.311 & 0.140 & 0.186 & 0.018 \\
0.4 & 0.393 & 0.234 & 0.240 & 0.033 \\
\hline \hline
\end{tabular}

${ }^{\mathrm{a}}$ Reference 9. 
$\stackrel{M^{2}}{\longrightarrow}$

(a)

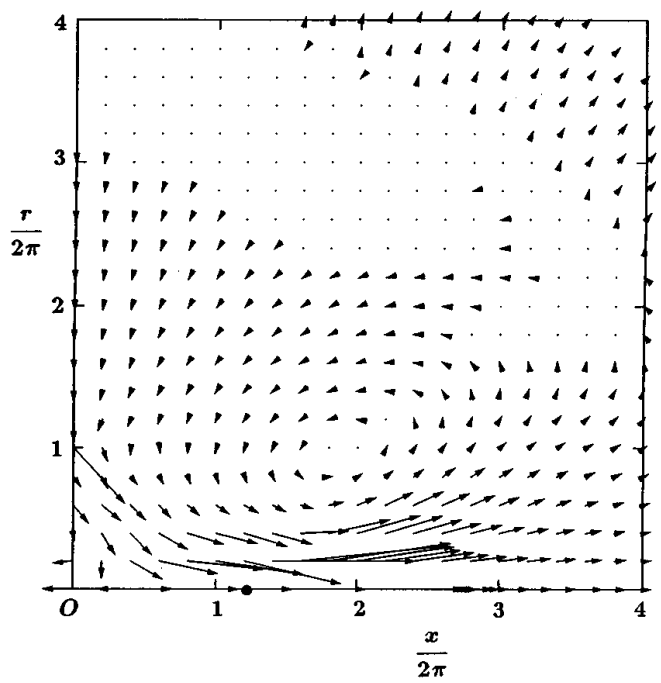

$\stackrel{M^{2}}{\longrightarrow}$

(b)

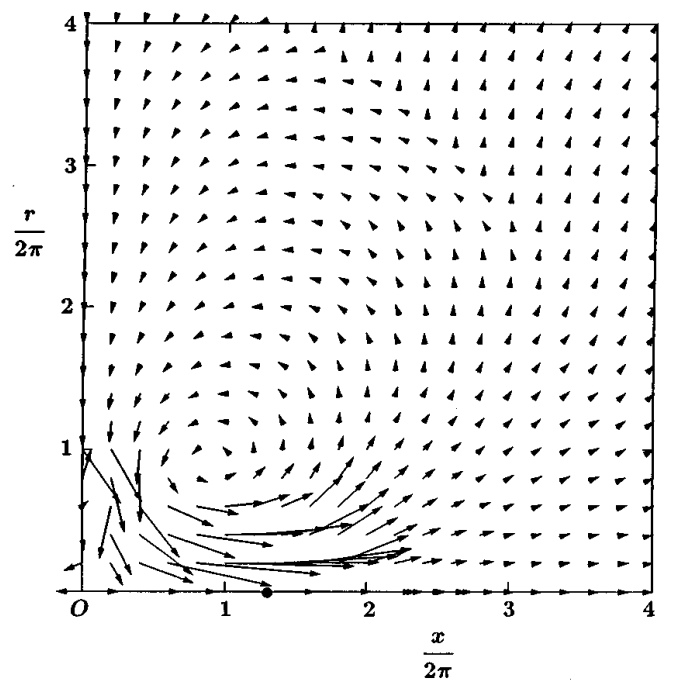

$\longrightarrow$

(c)

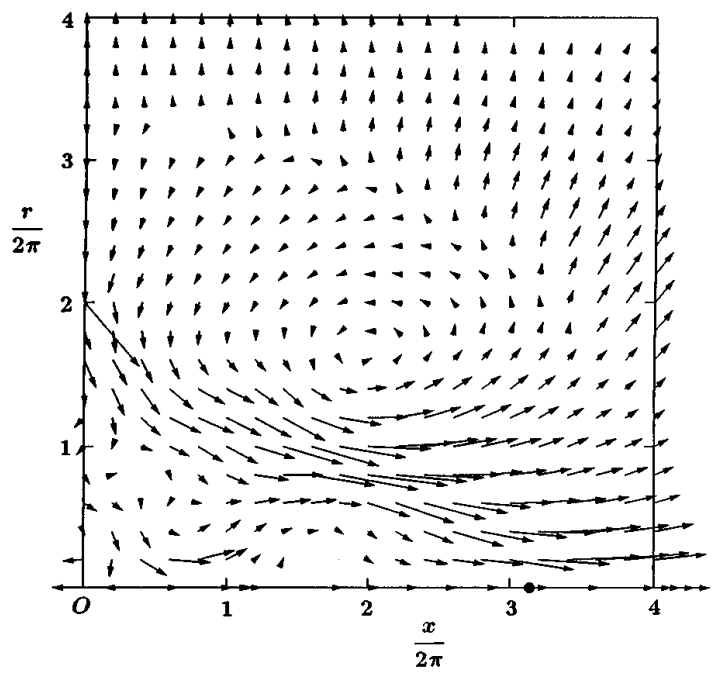

$\stackrel{M^{2}}{\longrightarrow}$

(d)

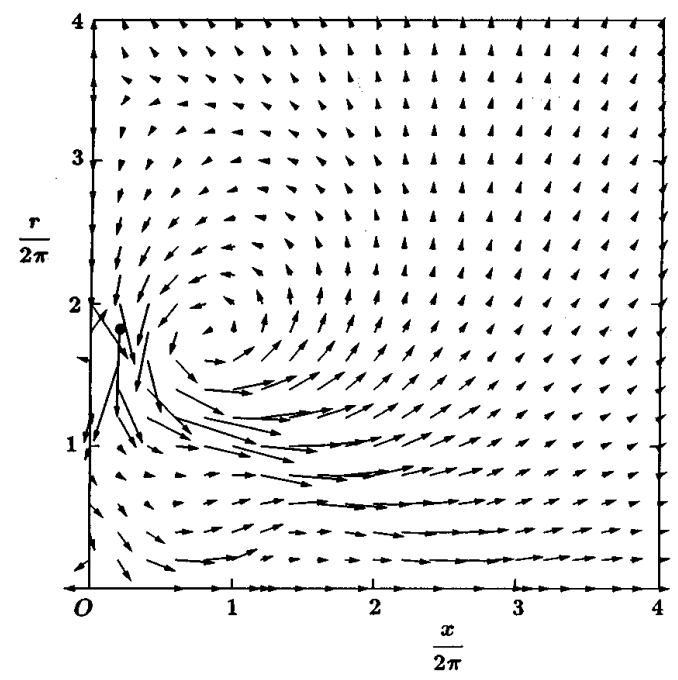

$M^{2}$

(e)

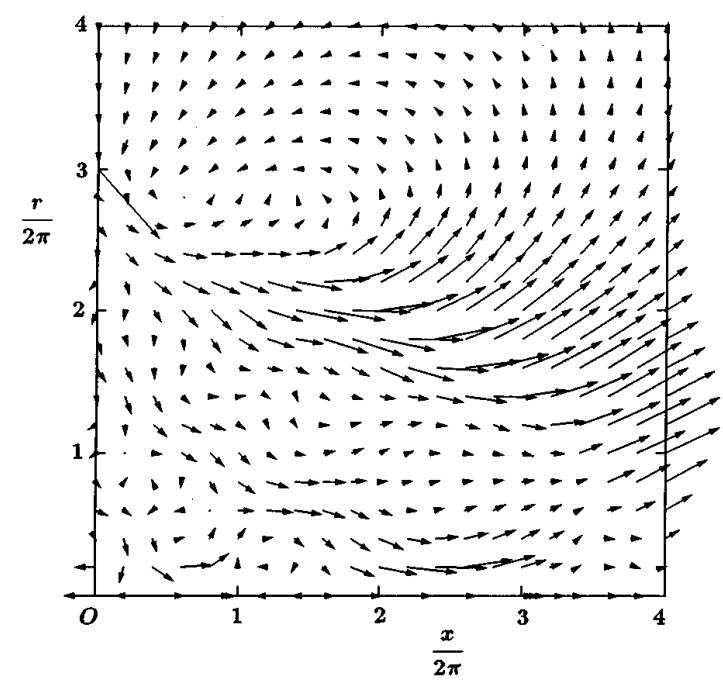

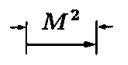

(f)

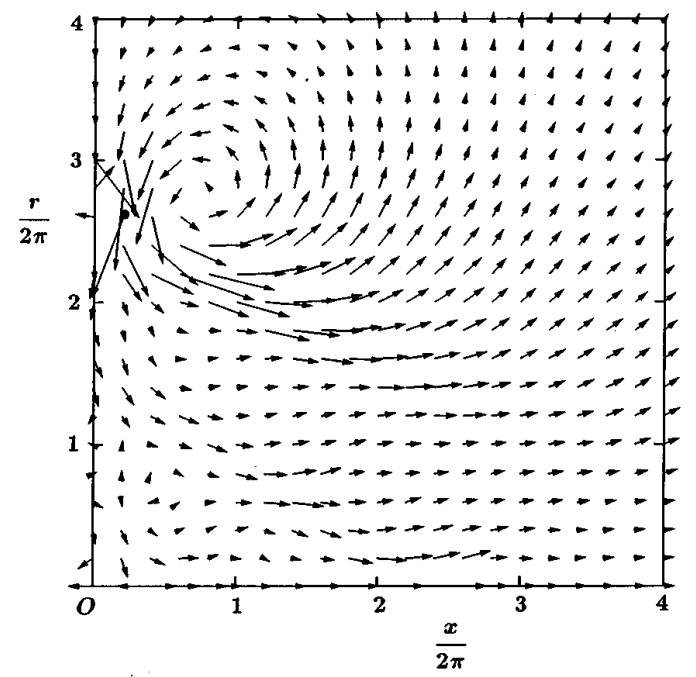

FIG. 12. Strongly nonlinear acoustic streaming. Arrows signify the time-averaged mass flux density vector $\mathbf{v}_{s}$. A reference arrow, the size of which corresponds to $\left|\mathbf{v}_{s} / M^{2}\right|=1$, is shown on the top of each figure. A closed circle signifies the point where $\left|\mathbf{v}_{s}\right|$ has the maximum value: (a) $R=1$ and $M=0.1$, (b) $R=1$ and $M=0.4$, (c) $R=2$ and $M=0.1$, (d) $R=2$ and $M=0.4$, (e) $R=3$ and $M=0.1$ and (f) $R=3$ and $M=0.4$. 

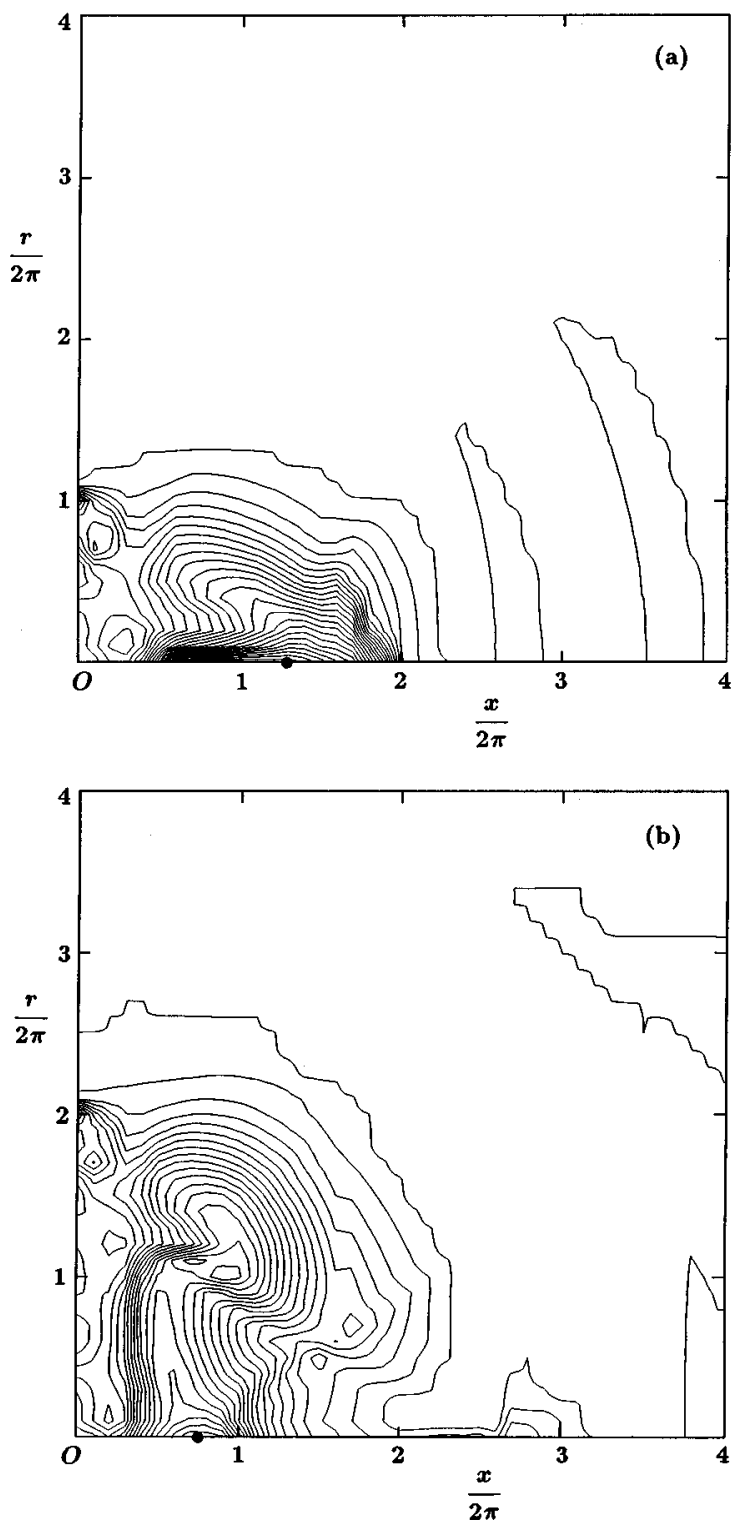

the axis, a new peak is produced at $x=25.8$ in the case of $R=2$, and two new peaks are produced at $x=19.6$ and 27.8 in the case of $R=3$ [cf. Fig. 2(c)].

In Fig. 7, the radial velocity in the case of $R=3$ and $M=0.4$ is plotted. It suggests that there is no singularity in the field, while in the linear case the radial velocity becomes infinity at the point $(x, r)=(0,2 \pi R)$ (see Fig. 3 and Sec. III C).

The pressure profile on the axis in the case of $R=2$ is compared with that of the linear wave with the same $R$ in Figs. 8(a) and 9(a), and is compared with that of the strongly nonlinear plane wave with the same $M$ in Figs. 8(b) and 9(b). In the case of $M=0.1$ and $R=2$, the nonlinear wave has positive spiky peaks (see Fig. 8). In the case of $M=0.4$ and $R=2$, the amplitude of the shock wave is reduced by the dissipation of energy at the shock front (see Fig. 9). The shock in the first wave cycle swallows the negative phase ahead of it, because the shock speed is larger than the sound speed. The same phenomenon has already been found in the

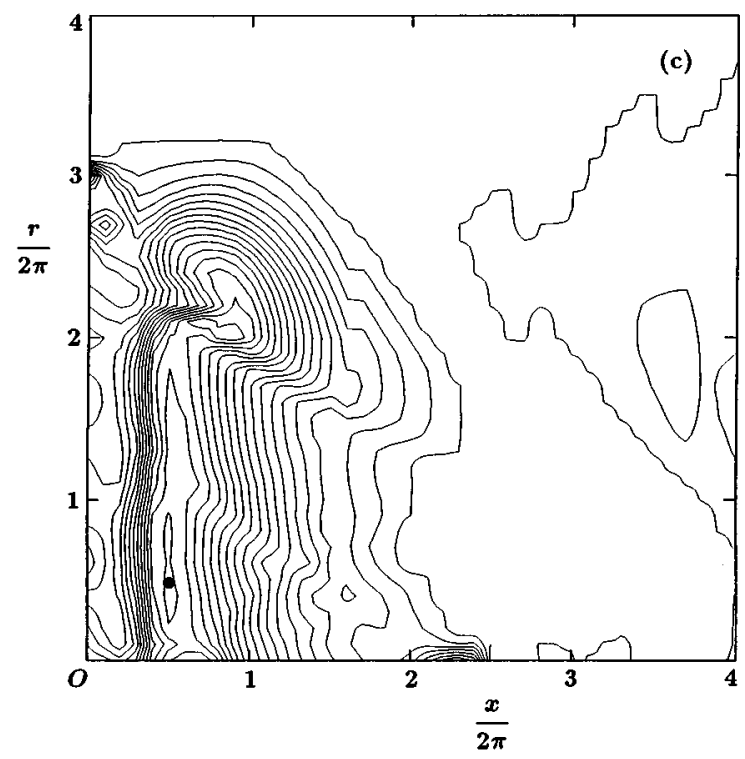

FIG. 13. Contour of the time-averaged density $\bar{\rho}$ at $t=12 \pi$ is plotted in steps of 0.01 in the neighborhood of the piston. A small closed circle denotes the point where the mean density is minimum: (a) $R=1$ and $M=0.4$. The minimum value is 0.795 at $(x, r)=(8.17,0)$. (b) $R=2$ and $M=0.4$. The minimum value is 0.816 at $(x, r)=(5.03,0)$. (c) $R=3$ and $M=0.4$. The minimum value is 0.816 at $(x, r)=(3.14,2.51)$.

problem of the strongly nonlinear plane waves. ${ }^{9}$ Figures 8 (b) and 9(b) display the strong nonlinearity of the wave on the axis, as compared with the plane wave with the same $M$.

Figure 10 shows the pressure contour, where the shock front is indicated by the contour lines distributed densely. Figure 11(a)-(c) are the magnifications of Fig. 10(b), (d), and (f), respectively. In these figures, in addition to shocks, the compression waves with steepened fronts are indicated by the folding contour lines, and sparse contour curves signify the expansion waves. The diffraction waves emanating from the edge of the source propagate "spherically," with being distorted by the nonlinear effect. As the wave approaches the axis, owing to the geometrical converging effect, the nonlinear effect becomes strong and hence the wave evolves into the shock. Consequently, the main shocks emitted from the piston face intersect with the shocks developed from the diffraction waves from the edge of source. That is, a multiple interference (intersection) of shock waves occurs (see Fig. 11). 

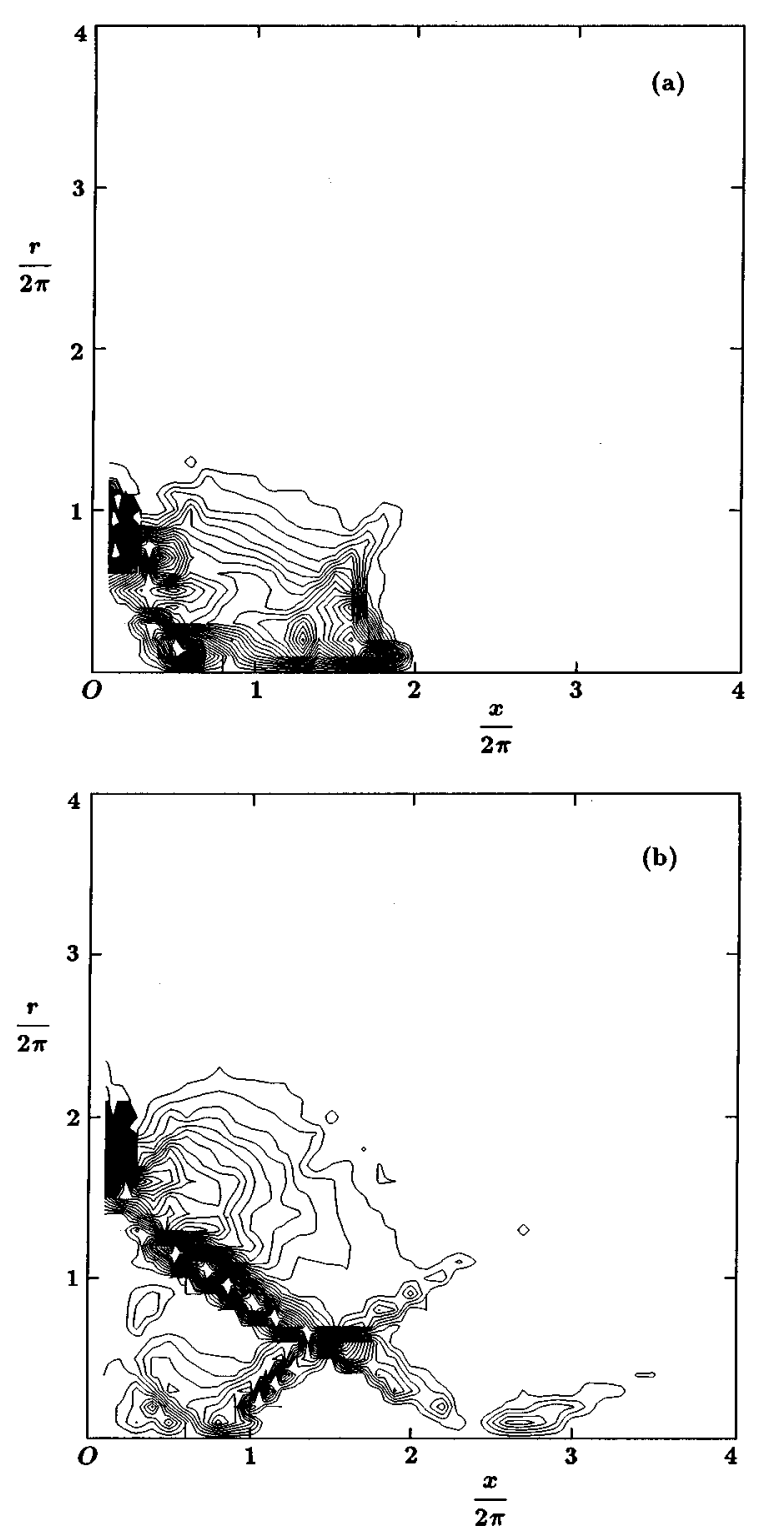

\section{B. Excitation of streaming and production of vorticity at the intersection of shocks}

Acoustic streaming is one of the most interesting phenomena in the propagation process of the strongly nonlinear waves. In the previous works of the strongly nonlinear plane wave ${ }^{9}$ and spherical wave, ${ }^{10}$ we have shown that streaming gradually rarefies the gas in the vicinity of the source. Note that in these problems streaming disappears in the weakly nonlinear limit in the near field (see Refs. 9 and 29).

In the strongly nonlinear case, contrary to the weakly nonlinear case treated in Sec. II, the mean mass flow $\mathbf{v}_{s}$ $=(\overline{\rho u}, \overline{\rho v})$ weakly depends on $T$ and $t$, because the wave motion is not in a steady state but in a quasi-steady state. ${ }^{9,10}$ The results presented in the following are calculated in terms of $T=2 \pi$, as in Refs. 9 and 10 .

Figure 12 shows that streaming forms a circulatory flow pattern in front of the piston face, which is a cross section of a vortex-ring-like flow, superposed on the fine structure of weakly nonlinear streaming (cf. Fig. 5). For a fixed $M$ and $R$, the location of the core of the vortex-ring-like flow does not

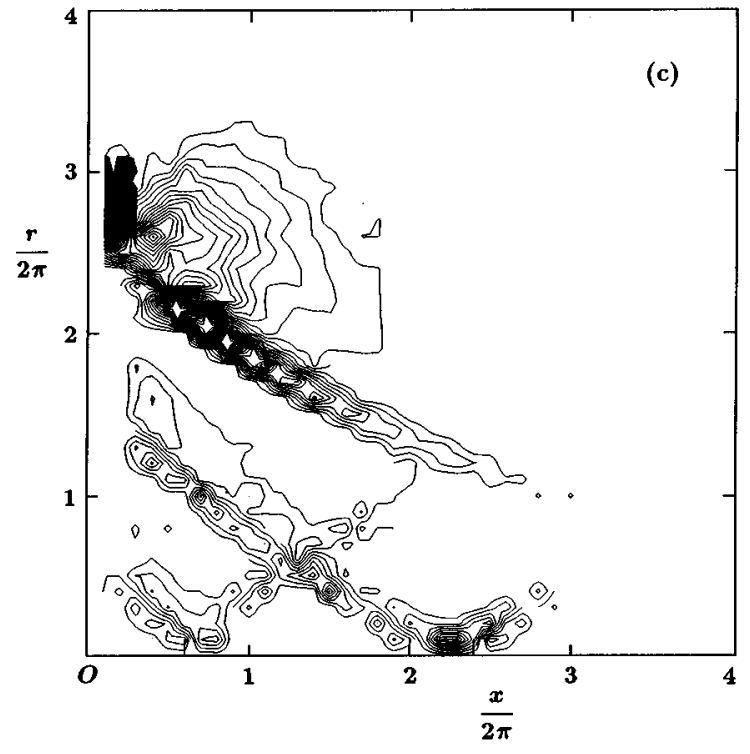

FIG. 14. Contour of the magnitude of time-averaged vorticity $\bar{\Omega}$ at $t=12 \pi$ is plotted in steps of 0.01 in the neighborhood of the piston: (a) $R=1$ and $M=0.4$. The maximum is 0.30 at $(1.26,5.65)$ and the minimum is -0.67 at $(1.26,4.40)$. (b) $R=2$ and $M=0.4$. The maximum is 0.43 at $(1.26,11.94)$ and the minimum is -0.52 at $(0.63,10.05)$. (c) $R=3$ and $M=0.4$. The maximum is 0.27 at $(1.26,18.22)$ and the minimum is -0.56 at $(0.63$, 16.34).

change for the duration of computation (from the time of formation of the vortex ring to the time $t=12 \pi$ ). With increase in $M$, the core of the vortex-ring-like flow approaches the edge of the piston. Note that, in the case of $M=0.1$ and $R=3$, the maximum value of $\left|\mathbf{v}_{s}\right|$ is attained at a point outside the frame of the graph, $(x, r)=(28.3,0)$. In Table I, the maximum value of $\left|\mathbf{v}_{s}\right|$ is compared with that of the strongly nonlinear plane wave. ${ }^{9}$ The streaming excited in the piston problem is much stronger than that in the plane wave problem, although the region where $\mathbf{v}_{s}$ is strong is small as shown in Fig. 12.

We also examine a time-averaged density of the gas defined as

$$
\bar{\rho}(x, r, t ; T)=\frac{1}{T} \int_{t-T}^{t} \rho(x, r, \tau) d \tau .
$$

As can be seen in Fig. 13, the gas in the neighborhood of the piston is rarefied by the streaming. Since the streaming is strong, the rarefaction of the gas occurs during several periods of oscillation of the piston; the rate of the rarefaction is 


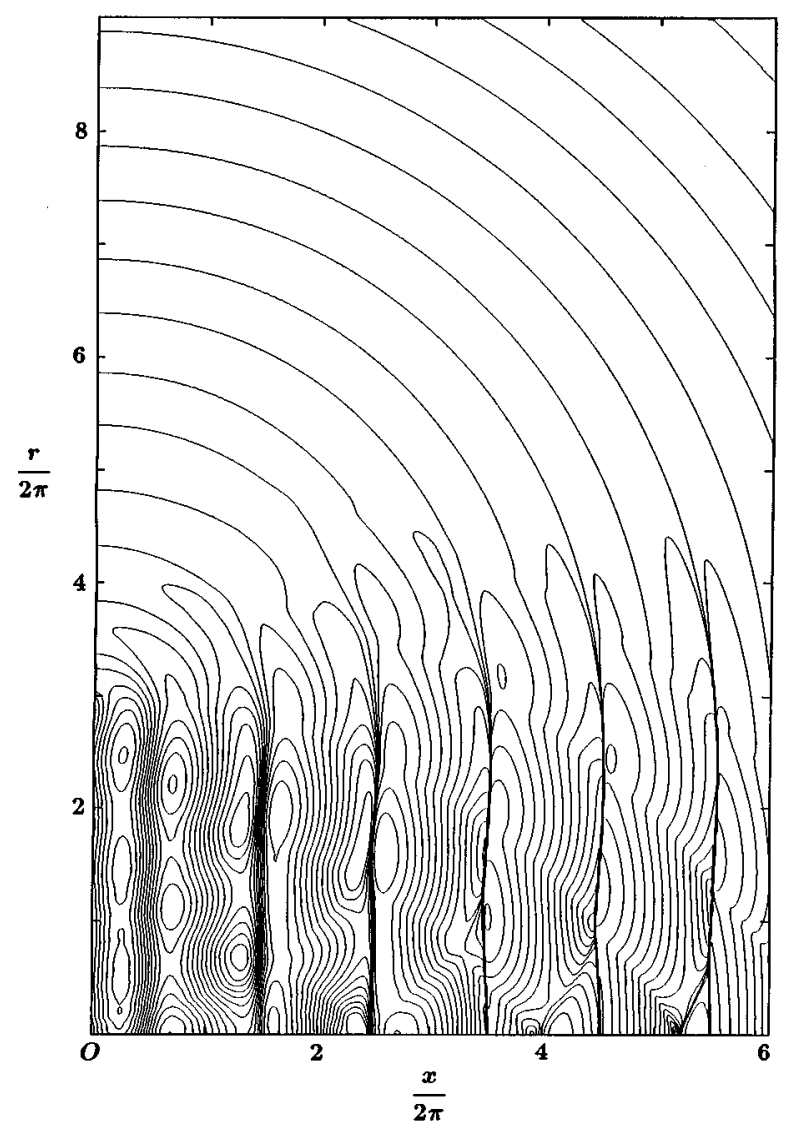

FIG. 15. Pressure contour in the case of $M=0.05$ and $R=3$ at $t=16 \pi$. The shock interference phenomenon almost disappears [cf. Fig. 10(f)].

almost twice as large as that in the plane wave problem. ${ }^{9}$

Even if the gas is initially uniform and at rest, and the viscosity of the gas is negligible except for the shock front, once a nonplanar shock wave of nonuniform strength is formed, the flow behind the shock may become rotational. In the case of a weak and steady shock, however, since the production of vorticity across the shock front is of the third order of the shock strength, the flow behind the shock can continue to be regarded as the irrotational one. ${ }^{30}$ However, in the problem concerned, the vorticity production at the shock front may not be negligible. In Fig. 14, we shall show the magnitude of time-averaged vorticity $\bar{\Omega}$ defined as

$$
\bar{\Omega}(x, r, t ; T)=\frac{1}{T} \int_{t-T}^{t}\left[\frac{\partial v}{\partial x}(x, r, \tau)-\frac{\partial u}{\partial r}(x, r, \tau)\right] d \tau .
$$

The vorticity is mainly produced at the intersection point of the shock from the piston face and the shock developed from the diffraction wave. Roughly speaking, owing to the assumption of $\operatorname{Re} \gg 1$, the generated vorticity is not diffused but convected and strengthened by local compression (or weakened by local expansion). ${ }^{31}$ Since the flow field can, to a very rough approximation, be regarded as periodic, the effects of convection and local compression and expansion may be cancelled by time average. As a result, the timeaveraged vorticity is localized in a narrow region along the locus of the intersection point of shocks. The narrow region of concentration of the vorticity corresponds to the region where the mean mass flow shown in Fig. 12 is strong.

In the strongly nonlinear case, as is seen from Fig. 12, streaming can be regarded as the superposition of a vortexring-like strong flow and a weak flow similar to that shown in Sec. II. The excitation of strong flow with intense rarefaction effect is attributed to the formation of moderately strong shocks which propagate with entraining the gas. Furthermore, the mutual interference of moderately strong shocks results in the production of vorticity which leads to the vortex-ring-like flow. In the weakly nonlinear case, the shock is too weak to entrain the gas appreciably, and hence the gas is not rarefied [at least to the approximation of $\left.O\left(M^{2}\right)\right]$. The shock interference does not occur because the nonlinear effect is too weak to transform a diffraction wave into a shock. Therefore, the vorticity produced at shock fronts is negligibly small. Accordingly, as the nonlinearity is reduced from the strongly nonlinear case, the vortex-ringlike flow disappears, while the weak streaming motion remains, whose generation mechanism is not relevant to any dissipation process (see, e.g., Ref. 15). We demonstrate in Figs. 15 and 16 that the shock interference is weakened and vortex-ring-like flow vanishes when the acoustic Mach number is reduced to $M=0.05$. Clearly, the numerical result for $M=0.05$ shown in Fig. 16 supports the validity of the weakly nonlinear analysis presented in the Appendices.

\section{Separation and generation of vortex at the edge}

During a half period from $t=2 n \pi$ to $t=(2 n+1) \pi$, the piston is drawn back from $x=0$ to $x=-2 M$, when the gas is sucked into the hole in the wall (see Fig. 1). At that time, the flow separates at the edge and a vortex is generated. The vectors of computed velocity near the edge of the source at $t=11 \pi$ are plotted in Fig. 17, where the isovorticity contours

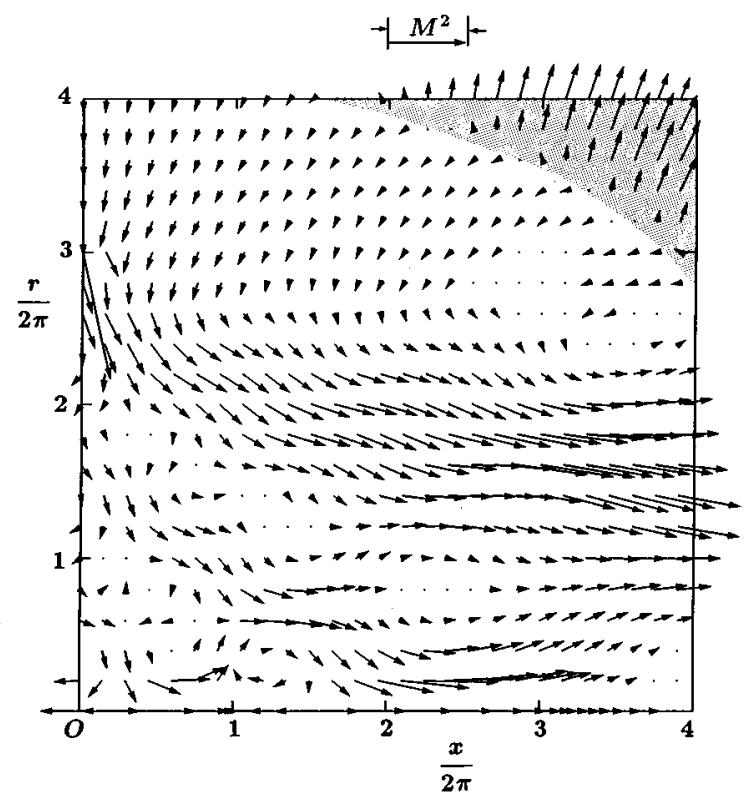

FIG. 16. Streaming without vortex-ring-like flow in the case of $M=0.05$ and $R=3$ at $t=16 \pi$. At this time, the initial transient effect still remains in the shadowed region [cf. Fig. 5(c)]. 


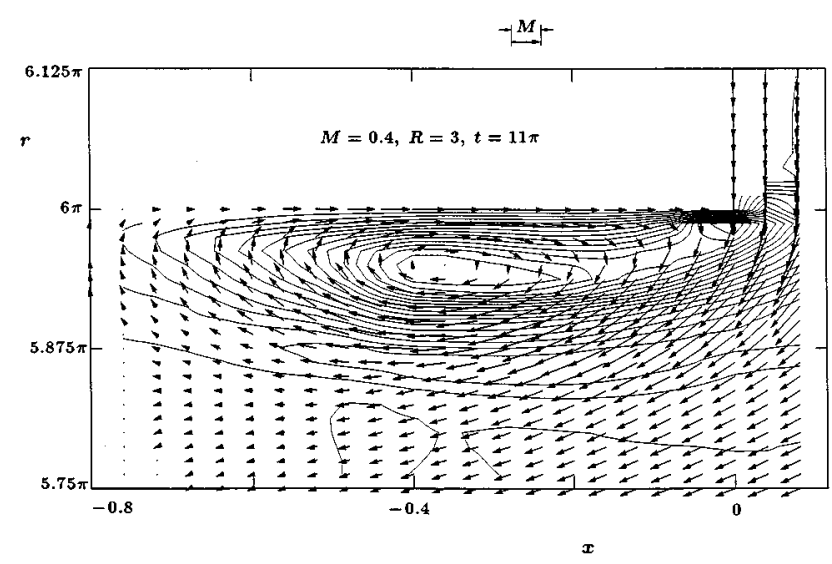

FIG. 17. Snapshot of the velocity field in the vicinity of the edge of the piston and the isovorticity contours at $t=11 \pi$, when the piston face is located at $x=-2 M=-0.8(M=0.4)$. The flow separates at the edge and the circulatory flow occurs.

are superimposed. The separation introduces a vortex sheet (the surface of tangential discontinuity) in the flow field (in Fig. 17, the vortex sheet is smeared out over mesh cells by the numerical dissipation). The vortex sheet rolls up into a vortex (circulatory flow). Note that we have not fixed the point of separation in any way and the circulating region develops spontaneously in the course of computation. The vorticity originated from the vortex sheet does not considerably affect the mean vorticity field outside the neighborhood of the source for the same reason as stated in Sec. III B (see Fig. 14).

\section{CONCLUSION}

In Sec. III, we have exhibited several interesting phenomena caused by the strongly nonlinear effect combined with the diffraction effect in the piston problem of $M=O(1), \operatorname{Re} \gg 1$, and $R=O(1)$, although the numerical result has been confined in the near field and in an initial stage of evolution of the wave. We have also revealed the property of weakly nonlinear acoustic streaming in Sec. II B. In the following, we shall briefly summarize the main results.

(i) The strongly nonlinear effect rapidly distorts the profile of the wave radiated directly from the source, and this leads to the shock formation near the source.

(ii) The strongly nonlinear effect also distorts the profile of the diffraction wave emanating from the edge of the piston, and the wave also evolves into the shock wave near the axis. Combined with (i), this leads to a multiple interference of shock waves.

(iii) Both the weakly and the strongly nonlinear waves excite acoustic streaming in the near field. In particular, the weakly nonlinear streaming is induced without any dissipation process.

(iv) Streaming produced in the case of $M=O(1)$ is considerably strong as compared with that in the strongly nonlinear plane waves with the same $M$. The strong streaming reduces a mean density of the gas in the vicinity of the piston, during the several periods of harmonic oscillation of the piston.
The strong streaming forms a vortex-ring-like flow pattern, in addition to a fine structure which appears in the weakly nonlinear streaming. The core of the vortex-ring-like flow approaches the piston face with increase in $M$.

(vi) The vorticity produced behind the shock is localized in a narrow region along the locus of the intersection point of shocks. The region of concentration of the vorticity corresponds to that where the mean mass flow is strong.

(vii) In the solution of the linear potential flow, the radial velocity diverges at the edge of the source, because the potential flow does not admit the generation of vortex sheet, i.e., separation. The numerical solution for the strongly nonlinear wave can make clear the feature of separation and formation of vortex at the edge.

\section{ACKNOWLEDGMENT}

This work was partially supported by The Kurata Research Grant from The Kurata Foundation.

\section{APPENDIX A: REFORMULATION OF THE PROBLEM IN THE WEAKLY NONLINEAR CASE}

The purposes of Appendices A-D are (i) to show that the well-known linear solution has a singularity at the edge of the source, i.e., the radial component of fluid velocity diverges at the edge, and (ii) to examine acoustic streaming in the weakly nonlinear case of $M \ll 1$ and Re $\gg 1$. The singularity is originated from the assumption of irrotational flow. In fact, the numerical solution presented in Sec. III demonstrates the generation of vorticity at the edge and the fluid velocity never diverges. In spite of the singularity, the linear solution is widely accepted to be plausible. In the following Appendices, we shall confirm that the linear solution may be regarded as the correct solution except for a small neighborhood of the edge.

For these purposes, we shall formulate the weakly nonlinear problem of $M \ll 1, \mathrm{Re} \gg 1$, and $R=O(1)$ in terms of the nondimensional velocity potential $\Phi(x, r, t)$, which is related to the normalized fluid velocity as

$$
u=\frac{\partial \Phi}{\partial x} \quad \text { and } \quad v=\frac{\partial \Phi}{\partial r} .
$$

The governing equation for $\Phi$ can be written in the form

$$
\begin{aligned}
\left(\frac{\partial^{2}}{\partial x^{2}}+\frac{\partial^{2}}{\partial r^{2}}+\frac{1}{r} \frac{\partial}{\partial r}-\frac{\partial^{2}}{\partial t^{2}}\right) \Phi= & \frac{\partial}{\partial t}\left[\left(\frac{\partial \Phi}{\partial x}\right)^{2}+\left(\frac{\partial \Phi}{\partial r}\right)^{2}\right. \\
& \left.+\frac{\gamma-1}{2}\left(\frac{\partial \Phi}{\partial t}\right)^{2}\right],
\end{aligned}
$$

where cubic and higher-order terms in $\Phi$ are neglected. The normalized pressure $p$ and density $\rho$ are then expressed as

$$
\begin{aligned}
(\gamma p)^{(\gamma-1) / \gamma}=\rho^{\gamma-1}= & 1-(\gamma-1)\left[\frac{\partial \Phi}{\partial t}+\frac{1}{2}\left(\frac{\partial \Phi}{\partial x}\right)^{2}\right. \\
& \left.+\frac{1}{2}\left(\frac{\partial \Phi}{\partial r}\right)^{2}\right] .
\end{aligned}
$$




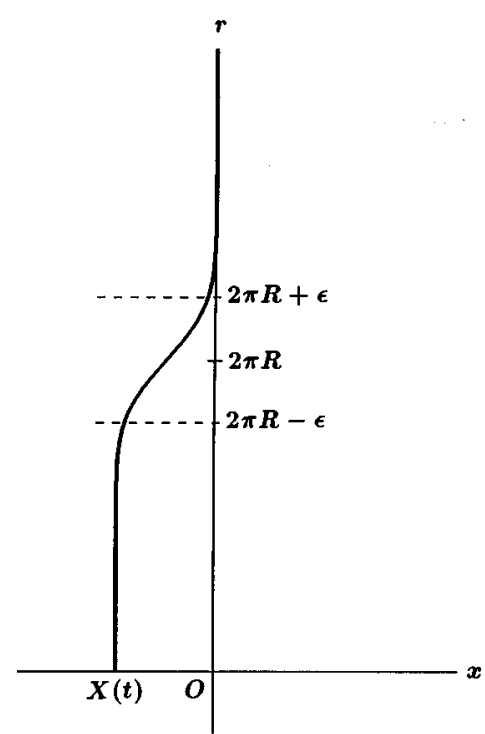

FIG. A1. Geometry of the smoothed boundary. The geometry shown in Fig. 1 can be obtained in the limit as $\epsilon \rightarrow 0$.

Note that the definitions of the variables (except for $\Phi$ ) are the same as those in the strongly nonlinear case given by Eq.

(2) in Sec. I.

The initial condition (7) in Sec. I can be rewritten into

$\Phi=\frac{\partial \Phi}{\partial t}=0 \quad$ at $\quad t=0$ for $x>0$.

We consider the geometry of the boundary prescribed by an equation

$$
x=\frac{M}{2}(\cos t-1) \operatorname{erfc}\left(\frac{r-2 \pi R}{\epsilon}\right) \quad(t>0),
$$

where $\epsilon$ is a nondimensional parameter sufficiently small compared with unity (see Fig. A1), and erfc denotes the complementary error function,

$$
\operatorname{erfc}(x)=1-\frac{2}{\sqrt{\pi}} \int_{0}^{x} e^{-\xi^{2}} d \xi
$$

Note that Eq. (A5) is reduced to Eq. (8) in Sec. I as $\epsilon \longrightarrow 0$. By using Eqs. (A1) and (A5), the boundary condition can be expressed as

$$
\begin{aligned}
\frac{\partial \Phi}{\partial x}= & -\frac{M}{2} \sin t \operatorname{erfc}\left(\frac{r-2 \pi R}{\epsilon}\right)-M(\cos t-1) \frac{1}{\epsilon \sqrt{\pi}} \\
& \times \exp \left[-\left(\frac{r-2 \pi R}{\epsilon}\right)^{2}\right] \frac{\partial \Phi}{\partial r} \\
& \text { at } \quad x=\frac{M}{2}(\cos t-1) \operatorname{erfc}\left(\frac{r-2 \pi R}{\epsilon}\right) \text { for } t>0 .
\end{aligned}
$$

We shall remark that, in the limit as $\epsilon \rightarrow 0$, Eq. (A6) differs from Eqs. (9) and (10) by a term including the Dirac delta function (see Appendix C). As shown in Appendices B and $\mathrm{C}$, the delta function term does not appear in the lowest order (linear approximation) and it is included in the second (and higher order) approximation. The divergence of $v$ at the edge is therefore not connected with it. If it were not for the delta function term, the mass conservation law could not be satisfied in the second order of approximation (see Appendix C). In other words, the difference of Eq. (A6) from the boundary conditions (9) and (10) is inevitable for obtaining the solution to the second order under the assumption of irrotational flow, which may be appropriate only outside a small region near the edge. If one takes another approach (the method of matched asymptotic expansions) that he separately treats the small region with a Kutta condition and matches this "inner solution" with an irrotational "outer solution," then the modified geometry described by Eq. (A5) is not necessary and the velocity may not diverge. However, in order to obtain a steady-state acoustic streaming valid outside the small region, we only require the first-order solution and the steady component of second-order velocity which are correct everywhere except for the small neighborhood of the edge. To this end, the present simple approach is sufficient.

In order to solve the problem formulated above, we shall expand the dependent variables in powers of $M$ :

$$
\left(\begin{array}{c}
\Phi \\
u \\
v \\
p-(1 / \gamma) \\
\rho-1
\end{array}\right)=M\left(\begin{array}{c}
\Phi_{1} \\
u_{1} \\
v_{1} \\
p_{1} \\
\rho_{1}
\end{array}\right)+M^{2}\left(\begin{array}{c}
\Phi_{2} \\
u_{2} \\
v_{2} \\
p_{2} \\
\rho_{2}
\end{array}\right)+O\left(M^{3}\right)
$$

Substituting expansion (A7) into Eqs. (A1)-(A6) and equating coefficients of like powers of $M$, we can obtain the successive systems of equations for $\Phi_{1}$ and $\Phi_{2}$ (see below).

\section{APPENDIX B: LINEAR SOLUTION}

In the leading order of approximation in the near field, the weakly nonlinear problem formulated in Appendix A is reduced to the initial- and boundary-value problem for the linear wave equation:

$$
\begin{aligned}
& \left(\frac{\partial^{2}}{\partial x^{2}}+\frac{\partial^{2}}{\partial r^{2}}+\frac{1}{r} \frac{\partial}{\partial r}-\frac{\partial^{2}}{\partial t^{2}}\right) \Phi_{1}=0, \\
& \left.\Phi_{1}\right|_{t=0}=\left.\frac{\partial \Phi_{1}}{\partial t}\right|_{t=0}=0 \quad(x>0), \\
& \left.\frac{\partial \Phi_{1}}{\partial x}\right|_{x=0}=-H(2 \pi R-r) H(r) \sin t \\
& \quad(t>0) \quad(\text { in the limit as } \epsilon \rightarrow 0) .
\end{aligned}
$$

The fundamental expression of the solution is the so-called Rayleigh surface integral,

$$
\Phi_{1}=\frac{1}{\pi} \int_{0}^{\pi} d \varphi \int_{0}^{2 \pi R} H(t-\mu) \frac{\sin (t-\mu) \sigma d \sigma}{\mu}
$$

where $\mu=\sqrt{x^{2}+r^{2}+\sigma^{2}-2 r \sigma \cos \varphi}$.

Introducing a new variable $\theta$,

$$
\theta=\arccos \left(\frac{\sigma \cos \varphi-r}{\sqrt{r^{2}+\sigma^{2}-2 r \sigma \cos \varphi}}\right),
$$

and changing the variables of integration $(\sigma, \varphi)$ to $(\mu, \theta)$, we can carried out the integral with respect to $\mu$. As a result, we 
obtain a useful representation: ${ }^{32}$ for $r<2 \pi R$,

$$
\begin{aligned}
\Phi_{1}= & \frac{1}{\pi} \int_{0}^{\pi} H\left(t-\mu_{M}\right)\left[\cos \left(t-\mu_{M}\right)-1\right] d \theta \\
& -H(t-x)[\cos (t-x)-1] ;
\end{aligned}
$$

and for $r \geqslant 2 \pi R$,

$$
\begin{aligned}
\Phi_{1}= & \frac{1}{\pi} \int_{\theta_{c}}^{\pi}\left\{H\left(t-\mu_{M}\right)\left[\cos \left(t-\mu_{M}\right)-1\right]\right. \\
& \left.-H\left(t-\mu_{m}\right)\left[\cos \left(t-\mu_{m}\right)-1\right]\right\} d \theta,
\end{aligned}
$$

where $\mu_{M}$ and $\mu_{m}$ are functions of $x, r$, and $\theta$, defined as

$$
\begin{aligned}
& \mu_{m}=\sqrt{x^{2}+\left[\sqrt{(2 \pi R)^{2}-(r \sin \theta)^{2}}+r \cos \theta\right]^{2}}, \\
& \mu_{M}=\sqrt{x^{2}+\left[\sqrt{(2 \pi R)^{2}-(r \sin \theta)^{2}}-r \cos \theta\right]^{2}},
\end{aligned}
$$

and $\theta_{c}$ is given by

$$
\theta_{c}=\pi-\arcsin (2 \pi R / r) .
$$

A significant physical interpretation for Eqs. (B6) and (B7) has been given in Ref. 26.

The pressure and the velocity components are then given as follows: for $r<2 \pi R$,

$$
\begin{aligned}
p_{1}= & \rho_{1}=-\frac{\partial \Phi_{1}}{\partial t}=\frac{1}{\pi} \int_{0}^{\pi} H\left(t-\mu_{M}\right) \sin \left(t-\mu_{M}\right) d \theta \\
& -H(t-x) \sin (t-x), \\
u_{1}= & \frac{\partial \Phi_{1}}{\partial x}=\frac{x}{\pi} \int_{0}^{\pi} H\left(t-\mu_{M}\right) \frac{\sin \left(t-\mu_{M}\right)}{\mu_{M}} d \theta \\
& -H(t-x) \sin (t-x), \\
v_{1}= & \frac{\partial \Phi_{1}}{\partial r}=\frac{1}{\pi} \int_{0}^{\pi} H\left(t-\mu_{M}\right) \alpha_{M} \sin \left(t-\mu_{M}\right) d \theta ;
\end{aligned}
$$

and for $r \geqslant 2 \pi R$,

$$
\begin{aligned}
p_{1}= & \rho_{1}=-\frac{\partial \Phi_{1}}{\partial t}=\frac{1}{\pi} \int_{\theta_{c}}^{\pi}\left[H\left(t-\mu_{M}\right) \sin \left(t-\mu_{M}\right)\right. \\
& \left.-H\left(t-\mu_{m}\right) \sin \left(t-\mu_{m}\right)\right] d \theta, \\
u_{1}= & \frac{\partial \Phi_{1}}{\partial x}=\frac{x}{\pi} \int_{\theta_{c}}^{\pi}\left[H\left(t-\mu_{M}\right) \frac{\sin \left(t-\mu_{M}\right)}{\mu_{M}}\right. \\
& \left.-H\left(t-\mu_{m}\right) \frac{\sin \left(t-\mu_{m}\right)}{\mu_{m}}\right] d \theta, \\
v_{1}= & \frac{\partial \Phi_{1}}{\partial r}=\frac{1}{\pi} \int_{\theta_{c}}^{\pi}\left[H\left(t-\mu_{M}\right) \alpha_{M} \sin \left(t-\mu_{M}\right)\right. \\
& \left.-H\left(t-\mu_{m}\right) \alpha_{m} \sin \left(t-\mu_{m}\right)\right] d \theta,
\end{aligned}
$$

where

$$
\begin{aligned}
\alpha_{M}=\frac{\partial \mu_{M}}{\partial r}= & \frac{1}{\mu_{M}}(r \cos 2 \theta \\
& \left.+\frac{\left[2(r \sin \theta)^{2}-(2 \pi R)^{2}\right] \cos \theta}{\sqrt{(2 \pi R)^{2}-(r \sin \theta)^{2}}}\right), \\
\alpha_{m}=\frac{\partial \mu_{m}}{\partial r}= & \frac{1}{\mu_{m}}(r \cos 2 \theta \\
& \left.-\frac{\left[2(r \sin \theta)^{2}-(2 \pi R)^{2}\right] \cos \theta}{\sqrt{(2 \pi R)^{2}-(r \sin \theta)^{2}}}\right) .
\end{aligned}
$$

Note that the Heaviside unit step function in the integrand is related to a transient effect caused by the initial condition (B2). If $t>\sqrt{x^{2}+(2 \pi R+r)^{2}}$ for a given field point $(x, r)$, then the Heaviside step function can be replaced by unity. That is, the phenomenon at the point becomes periodic for $t>\sqrt{x^{2}+(2 \pi R+r)^{2}}$.

As is readily verified, $\Phi_{1}$ and $p_{1}$ (and also $\rho_{1}$ ) are continuous at $r=2 \pi R$. However, the axial velocity component $u_{1}$ is discontinuous at the edge of the piston, i.e., $x=0$ and $r=2 \pi R$, because boundary condition (B3) is in itself discontinuous. Furthermore, we can show that the radial component $v_{1}$ has a logarithmic singularity at the edge, namely,

$$
\begin{aligned}
v_{1}(x, 2 \pi R)= & \left(V_{s}+\frac{1}{4 \pi^{2} R} \ln x\right) \sin t+V_{c} \cos t \\
& +o(1) \text { as } x \rightarrow 0 .
\end{aligned}
$$

Here, $V_{s}$ and $V_{c}$ are constants depending on $R$, defined as

$$
\begin{aligned}
& V_{s}=\frac{2}{\pi}-\mathbf{H}_{1}(4 \pi R)+2 R_{2} F_{3}\left(1,1 ; \frac{3}{2}, \frac{3}{2}, 2 ;-4 \pi^{2} R^{2}\right), \\
& V_{c}=\frac{1}{2} \int_{0}^{4 \pi R} J_{0}(z) d z-J_{1}(4 \pi R),
\end{aligned}
$$

where $\mathbf{H}_{1}$ is the Struve function of the first order, $J_{n}$ is the Bessel function of $n$th order, and ${ }_{2} F_{3}$ signifies a generalized hypergeometric function. ${ }^{33}$ In deriving Eqs. (B19)-(B21), we have used Eq. (B16) and have assumed $t>4 \pi R$, for simplicity.

The solution for $v_{1}$ thus becomes invalid at the edge of the piston. However, $v_{1}$ remains of $O(1)$ outside a small region including the singular point, because the divergence of logarithm is very slow (cf. Fig. 3).

\section{APPENDIX C: TIME-INDEPENDENT PART OF THE SECOND-ORDER SOLUTION}

We suppose that $t$ is sufficiently large so that the steadystate wave motion is realized in the near field. The secondorder velocity potential $\Phi_{2}$ may then consist of a timeindependent part $\bar{\Phi}_{2}$ and the second harmonic. Clearly, the time-independent part is governed by Laplace equation

$$
\frac{\partial^{2} \bar{\Phi}_{2}}{\partial x^{2}}+\frac{\partial^{2} \bar{\Phi}_{2}}{\partial r^{2}}+\frac{1}{r} \frac{\partial \bar{\Phi}_{2}}{\partial r}=0 .
$$


Substituting expansion (A7) into Eq. (A6) yields the boundary condition for $\Phi_{2}$,

$$
\begin{aligned}
\left.\frac{\partial \Phi_{2}}{\partial x}\right|_{x=0}= & -\left.H(2 \pi R-r) H(r)(\cos t-1) \frac{\partial u_{1}}{\partial x}\right|_{x=0} \\
& -\left.\delta(r-2 \pi R)(\cos t-1) v_{1}\right|_{x=0}
\end{aligned}
$$

(in the limit as $\epsilon \rightarrow 0$ ),

where $\delta(r)$ is the Dirac delta function. Substituting Eqs. (B12) and (B13) into the right-hand side of Eq. (C2) and averaging the result with respect to $t$, we obtain the boundary condition for the time-independent part $\bar{\Phi}_{2}$,

$$
\begin{aligned}
\left.\frac{\partial \bar{\Phi}_{2}}{\partial x}\right|_{x=0}= & H(2 \pi R-r) H(r)\left(\frac{1}{2 \pi} \int_{0}^{\pi} \frac{\sin \nu_{M}}{\nu_{M}} d \theta-\frac{1}{2}\right) \\
& -\frac{1}{2} V_{c} \delta(r-2 \pi R) \\
\equiv & f(r)-\frac{1}{2} V_{c} \delta(r-2 \pi R),
\end{aligned}
$$

where $\nu_{M}$ is the function of $r$ and $\theta$,

$$
\nu_{M}=\sqrt{(2 \pi R)^{2}-(r \sin \theta)^{2}}-r \cos \theta,
$$

and $V_{c}$ is the constant given by Eq. (B21) in Appendix B.

Using the method of Green's function gives the solution of Laplace equation as

$$
\bar{\Phi}_{2}=-\left.\frac{1}{\pi} \int_{0}^{\pi} d \varphi \int_{0}^{\infty} \frac{\partial \bar{\Phi}_{2}}{\partial x}\right|_{x=0} \frac{\sigma d \sigma}{\sqrt{x^{2}+r^{2}+\sigma^{2}-2 r \sigma \cos \varphi}} .
$$

Substituting Eq. (C3) into Eq. (C5), we have

$$
\begin{aligned}
\bar{\Phi}_{2}= & -\frac{2}{\pi} \int_{0}^{2 \pi R} \frac{\sigma f(\sigma)}{\sqrt{x^{2}+(r+\sigma)^{2}}} K\left[\frac{4 \sigma r}{x^{2}+(r+\sigma)^{2}}\right] d \sigma \\
& +\frac{2 R V_{c}}{\sqrt{x^{2}+(r+2 \pi R)^{2}}} K\left[\frac{8 \pi R r}{x^{2}+(r+2 \pi R)^{2}}\right],
\end{aligned}
$$

where $K[m]$ is the complete elliptic integral of the first kind,

$$
K[m]=\int_{0}^{\pi / 2} \frac{d \psi}{\sqrt{1-m \sin ^{2} \psi}} .
$$

Differentiating $\bar{\Phi}_{2}$ with respect to $x$ and $r$, we obtain the steady components of the second-order fluid velocity as follows:

$$
\begin{aligned}
\bar{u}_{2}= & \frac{\partial \bar{\Phi}_{2}}{\partial x}=\frac{2 x}{\pi} \int_{0}^{2 \pi R} \frac{\sigma f(\sigma)}{\sqrt{x^{2}+(r+\sigma)^{2}}\left[x^{2}+(r-\sigma)^{2}\right]} \\
& \times E\left[\frac{4 \sigma r}{x^{2}+(r+\sigma)^{2}}\right] d \sigma \\
& -\frac{2 R V_{c} x}{\sqrt{x^{2}+(r+2 \pi R)^{2}}\left[x^{2}+(r-2 \pi R)^{2}\right]} \\
& \times E\left[\frac{8 \pi R r}{x^{2}+(r+2 \pi R)^{2}}\right],
\end{aligned}
$$

$$
\begin{aligned}
\bar{v}_{2}= & \frac{\partial \bar{\Phi}_{2}}{\partial r}=\frac{1}{\pi r} \int_{0}^{2 \pi R} \frac{\sigma f(\sigma)}{\sqrt{x^{2}+(r+\sigma)^{2}}}\left\{K\left[\frac{4 \sigma r}{x^{2}+(r+\sigma)^{2}}\right]\right. \\
& \left.+\frac{r^{2}-x^{2}-\sigma^{2}}{x^{2}+(r-\sigma)^{2}} E\left[\frac{4 \sigma r}{x^{2}+(r+\sigma)^{2}}\right]\right\} d \sigma \\
& -\frac{R V_{c}}{r \sqrt{x^{2}+(r+2 \pi R)^{2}}}\left\{K\left[\frac{8 \pi R r}{x^{2}+(r+2 \pi R)^{2}}\right]\right. \\
& \left.+\frac{r^{2}-x^{2}-(2 \pi R)^{2}}{x^{2}+(r-2 \pi R)^{2}} E\left[\frac{8 \pi R r}{x^{2}+(r+2 \pi R)^{2}}\right]\right\}, \quad \text { (C9) }
\end{aligned}
$$

where $E[m]$ is the complete elliptic integral of the second kind,

$$
E[m]=\int_{0}^{\pi / 2} \sqrt{1-m \sin ^{2} \psi} d \psi .
$$

Clearly, the mass conservation law requires that the net mean mass flow across the $x=0$ plane must be zero, i.e.,

$$
\begin{aligned}
\left.\int_{0}^{\infty}\left(\overline{\rho_{1} u_{1}}+\overline{u_{2}}\right)\right|_{x=0} r d r & =\left.\int_{0}^{2 \pi R}\left(\overline{\rho_{1} u_{1}}+\overline{u_{2}}\right)\right|_{x=0} r d r \\
& =0,
\end{aligned}
$$

although $\overline{\rho_{1} u_{1}}+\overline{u_{2}}$ is not necessarily zero at each point on the surface $x=0$ and $0 \leqslant r \leqslant 2 \pi R$ (cf. Fig. 5), because it is a mean surface of the oscillating piston face. By the detailed numerical analysis (see Appendix D), we have confirmed that Eq. (C11) is satisfied and that the vector $\mathbf{v}_{s}=M^{2}\left(\overline{\rho_{1} u_{1}}\right.$ $\left.+\overline{u_{2}}, \overline{\rho_{1} v_{1}}+\overline{v_{2}}\right)$ is divergence-free throughout the near field. That is, the mass conservation law holds to the approximation of $O\left(M^{2}\right)$ [if the delta function term is not included in Eq. (C3), Eq. (C11) is not satisfied].

The time-independent components of the second-order velocity, $\bar{u}_{2}$ and $\bar{v}_{2}$, diverge at the edge of the piston. However, as in the case of $v_{1}$, the divergence of $\mathbf{v}_{s}$ is slow and the result shown in Fig. 5 may be valid except for the singular point (we have numerically confirmed that $\overline{u_{2}}$ and $\overline{v_{2}}$ diverge logarithmically as $x$ approaches 0 with $r=2 \pi R$ being fixed).

\section{APPENDIX D: EXPLICIT REPRESENTATIONS FOR $\overline{\rho_{1} u_{1}}$ and $\overline{\rho_{1} v_{1}}$}

The explicit representations for $\overline{\rho_{1} u_{1}}$ and $\overline{\rho_{1} v_{1}}$ are given as follows: for $r<2 \pi R$,

$$
\begin{aligned}
\overline{\rho_{1} u_{1}}= & \frac{1}{2}\left(\frac{1}{\pi} \int_{0}^{\pi} \cos \mu_{M} d \theta-\cos x\right) \\
& \times\left(\frac{x}{\pi} \int_{0}^{\pi} \frac{\cos \mu_{M}}{\mu_{M}} d \theta-\cos x\right) \\
& +\frac{1}{2}\left(\frac{1}{\pi} \int_{0}^{\pi} \sin \mu_{M} d \theta-\sin x\right) \\
& \times\left(\frac{x}{\pi} \int_{0}^{\pi} \frac{\sin \mu_{M}}{\mu_{M}} d \theta-\sin x\right),
\end{aligned}
$$




$$
\begin{aligned}
\overline{\rho_{1} v_{1}}= & \frac{1}{2 \pi}\left(\frac{1}{\pi} \int_{0}^{\pi} \cos \mu_{M} d \theta-\cos x\right) \\
& \times\left(\int_{0}^{\pi} \alpha_{M} \cos \mu_{M} d \theta\right) \\
& +\frac{1}{2 \pi}\left(\frac{1}{\pi} \int_{0}^{\pi} \sin \mu_{M} d \theta-\sin x\right) \\
& \times\left(\int_{0}^{\pi} \alpha_{M} \sin \mu_{M} d \theta\right) ;
\end{aligned}
$$

for $r>2 \pi R$,

$$
\begin{aligned}
\overline{\rho_{1} u_{1}}= & \frac{x}{2 \pi^{2}} \int_{\theta_{c}}^{\pi}\left(\cos \mu_{M}-\cos \mu_{m}\right) d \theta \int_{\theta_{c}}^{\pi}\left(\frac{\cos \mu_{M}}{\mu_{M}}\right. \\
& \left.-\frac{\cos \mu_{m}}{\mu_{m}}\right) d \theta+\frac{x}{2 \pi^{2}} \int_{\theta_{c}}^{\pi}\left(\sin \mu_{M}\right. \\
& \left.-\sin \mu_{m}\right) d \theta \int_{\theta_{c}}^{\pi}\left(\frac{\sin \mu_{M}}{\mu_{M}}-\frac{\sin \mu_{m}}{\mu_{m}}\right) d \theta, \\
\overline{\rho_{1} v_{1}}= & \frac{1}{2 \pi^{2}} \int_{\theta_{c}}^{\pi}\left(\cos \mu_{M}-\cos \mu_{m}\right) d \theta \int_{\theta_{c}}^{\pi}\left(\alpha_{M} \cos \mu_{M}\right. \\
& \left.-\alpha_{m} \cos \mu_{m}\right) d \theta+\frac{1}{2 \pi^{2}} \int_{\theta_{c}}^{\pi}\left(\sin \mu_{M}\right. \\
& \left.-\sin \mu_{m}\right) d \theta \int_{\theta_{c}}^{\pi}\left(\alpha_{M} \sin \mu_{M}-\alpha_{m} \sin \mu_{m}\right) d \theta
\end{aligned}
$$

where $\mu_{M}, \mu_{m}$, and $\theta_{c}$ are defined by Eqs. (B8)-(B10) in Appendix $\mathrm{B}$, and it is assumed that $t$ is sufficiently large.

\section{APPENDIX E: SUPPLEMENT TO THE NUMERICAL METHOD}

The numerical approach adopted in this paper is based on an explicit upwind finite difference scheme of the thirdorder accuracy in space and the first-order accuracy in time, ${ }^{11,12}$ which is known as one of the most successful and reliable schemes that has the capability of calculating the solutions for complicated flow field including discontinuous shocks. We do not need to give the algorithm to construct the scheme in this paper, since it is formulated in Refs. 11 and 12 at some length. In the following, we shall briefly describe the numerical implementation for the present problem (see Ref. 10 for detail of the treatment of boundary condition).

As noted in Ref. 11, upwind schemes have a tendency to give a smeared numerical result for the discontinuities (shocks) rapidly moving relative to the spatial coordinate of the mesh of computation. In order to obtain the high resolution for shocks, we shall introduce the new "spatial" coordinate $\xi=x-t$, which moves with the normalized sound speed in the positive $x$ direction, because in the present problem the shock speed is comparable with the speed of sound in the initial undisturbed state, $c_{0}$. Hence, the system (3)-(6) is rewritten into

$$
\frac{\partial w}{\partial t}+\frac{\partial f}{\partial \xi}+\frac{\partial g}{\partial r}=-\frac{h}{r},
$$

where

$$
\begin{aligned}
& w=\left(\begin{array}{c}
\rho \\
\rho u \\
\rho v \\
E_{t}
\end{array}\right), \quad f=\left(\begin{array}{c}
\rho u-\rho \\
\rho u^{2}+p-\rho u \\
\rho u v-\rho v \\
\left(E_{t}+p\right) u-E_{t}
\end{array}\right), \\
& g=\left(\begin{array}{c}
\rho v \\
\rho u v \\
\rho v^{2}+p \\
\left(E_{t}+p\right) v
\end{array}\right), \quad h=\left(\begin{array}{c}
\rho v \\
\rho u v \\
\rho v^{2} \\
\left(E_{t}+p\right) v
\end{array}\right) .
\end{aligned}
$$

The left-hand side of Eq. (E1) is discretized by using the high-resolution upwind finite difference scheme for spatially two-dimensional flow. The right-hand side is evaluated at every mesh point, as is usually done for the inhomogeneous conservation laws. A mesh of constant intervals, $\Delta t, \Delta \xi$, and $\Delta r$, is employed, for simplicity of construction of the scheme. The time step $\Delta t$ is taken to be sufficiently small so as to stabilize the computation, typically $\Delta t / \Delta \xi=\Delta t / \Delta r$ $=0.05$. The numerical results presented in Sec. III are, except for Figs. 15-17, computed with $\Delta \xi=2 \pi / 120$ and $\Delta r$ $=2 \pi / 120$. Figures $15-17$ are the results of computation with $\Delta \xi=2 \pi / 160$ and $\Delta r=2 \pi / 160$.

Since the constant intervals, $\Delta t, \Delta r$, and $\Delta \xi$, are used, the boundary, namely, the surface composed of the piston and the wall, is not always coincident with a mesh point $(k \Delta \xi, j \Delta r, n \Delta t)$, where $k, j$, and $n$ are integers (see Ref. 10). We therefore apply the quadratic interpolation to determine the velocity at the nearest mesh point to the boundary. On the other hand, to determine the density and pressure on the boundary and at the nearest point to the boundary, we use the quadratic extrapolation using the values at inner mesh points. On the axis, where $j=0, v$ is set to be zero, and the other quantities, $p, \rho$, and $u$, are interpolated by using symmetry relations. In the region of computation, there exist two rightangled corners, i.e., $(x, r)=(0,2 \pi R)$ and $(x, r)=(X(t), 2 \pi R)$ (cf. Fig. 1). In order to determine $p, \rho, u$, and $v$ at these points, we apply the quadratic interpolation using adjacent points.

The region of computation in $(x, r)$ plane is a rectangle larger than $t \times(t+2 \pi R)$ at time $t$ (maximum mesh size is larger than $960 \times 1440$ at $t=12 \pi$ for the case of $\Delta \xi=2 \pi / 160$ and $\Delta r=2 \pi / 160$ and $R=3$ ). As a result, we need not introduce any artificial (nonreflecting) boundary condition at the outer boundary of region of computation.

For a test of the numerical method, we have calculated a weakly nonlinear case of $M=0.01$ and compared the result normalized by $M$ with the linear solution, because, as long as we confine ourselves in a region near the piston, the weakly nonlinear wave behaves as the linear wave. The error is less than $3 \%$ almost everywhere in the vicinity of the piston except for the neighborhood of the edge when a mesh of $\Delta \xi$ $=\Delta r=2 \pi / 120$ is used.

In order to obtain the correct results for the mean mass flow, we have carried out the computations carefully, con- 
firming that the whole mass of the gas from the boundary to the head of the wave is conserved within a permissible numerical error.

${ }^{1}$ E. A. Zabolotskaya and R. V. Khokhlov, "Quasi-plane waves in the nonlinear acoustics of confined beams," Sov. Phys. Acoust. 15, 35-40 (1969).

${ }^{2}$ J. C. Lockwood, T. G. Muir, and D. T. Blackstock, "Directive harmonic generation in the radiation field of a circular piston," J. Acoust. Soc. Am. 53, 1148-1153 (1973).

${ }^{3}$ O. V. Rudenko and S. I. Soluyan, Theoretical Foundations of Nonlinear Acoustics (Consultants Bureau, New York, 1977), Chap. 9.

${ }^{4}$ S. I. Aanonsen, T. Barkve, J. N. Tjøtta, and S. Tjøtta, "Distortion and harmonic generation in the nearfield of a finite amplitude sound beam," J. Acoust. Soc. Am. 75, 749-768 (1984).

${ }^{5}$ M. F. Hamilton, J. N. Tjøtta, and S. Tjøtta, "Nonlinear effects in the farfield of a directive sound source,"' J. Acoust. Soc. Am. 78, 202-216 (1985).

${ }^{6}$ The diffusivity of sound, $\delta^{*}$, is defined as $\delta^{*}=\nu[(4 / 3)+(\zeta / \eta)+(\gamma-1) \kappa /$ $\eta c_{p}$ ], where $\nu=\eta / \rho_{0}$ is the kinematic viscosity, $\rho_{0}$ is the density in an initial undisturbed gas, $\eta$ is the viscosity, $\zeta$ is the bulk viscosity, $\gamma$ is the ratio of specific heats, $\kappa$ is the thermal conductivity, and $c_{p}$ is the specific heat for the ideal gas at constant pressure. See M. J. Lighthill, "Viscosity effects in sound waves of finite amplitude," in Surveys in Mechanics, edited by G. K. Batchelor and R. M. Davies (Cambridge U.P., Cambridge, 1956), pp. 250-351.

${ }^{7}$ Strictly speaking, this condition signifies the limit as $\mathrm{Re} \rightarrow \infty$, mathematically.

${ }^{8}$ In the theory of the weakly nonlinear waves, the term "near field" refers to a region whose distances from the source are comparable with a typical wavelength. However, in problems of large transducer of $R \gg 1$, the term has often been used for a region whose distances from the source are a few source radii, although, in the present case of $R=O(1)$, the latter definition is equivalent to the former.

${ }^{9}$ Y. Inoue and T. Yano, "Propagation of strongly nonlinear plane waves," J. Acoust. Soc. Am. 94, 1632-1642 (1993). See also Y. Inoue and T. Yano, "Propagation of acoustic shock waves of large amplitude," in Frontiers of Nonlinear Acoustics 12th ISNA, edited by M. F. Hamilton and D. T. Blackstock (Elsevier, London, 1990), pp. 141-146.

${ }^{10} \mathrm{~T}$. Yano and Y. Inoue, "Numerical study of strongly nonlinear acoustic waves, shock waves, and streaming caused by a harmonically pulsating sphere,' Phys. Fluids 6, 2831-2844 (1994).

${ }^{11} \mathrm{~S}$. Osher and F. Solomon, "Upwind difference schemes for hyperbolic systems of conservation laws," Math. Comput. 38, 339-374 (1982).

${ }^{12} \mathrm{~S}$. R. Chakravarthy and S. Osher, "Computing with high-resolution upwind schemes for hyperbolic equations," in Large-Scale Computations in Fluid Mechanics, edited by B. Engquist, S. Osher, and R. C. J. Somerville (American Mathematical Society, Providence, RI, 1985), pp. 57-86.

${ }^{13} \mathrm{Y}$. Inoue and T. Okigami, "Propagation of finite amplitude sound waves radiated from an oscillating rigid sphere,' J. Sound Vib. 118, 199-207 (1987).

${ }^{14} \mathrm{~T}$. Yano and Y. Inoue, "'Second order solution for non-linear sound waves radiated from an oscillating rigid sphere,' J. Sound Vib. 135, 385-397 (1989).

${ }^{15} \mathrm{~S}$. Furuyama and Y. Inoue, "Weakly nonlinear waves and acoustic streaming produced by an oscillating rigid cylinder," J. Acoust. Soc. Am. 95, 708-717 (1994)

${ }^{16} \mathrm{~S}$. Furuyama and Y. Inoue, "Weakly nonlinear waves and acoustic streaming produced by a vibrating strip flush with a plane wall,' J. Acoust. Soc. Am. 98, 3443-3455 (1995).

${ }^{17}$ M. J. Lighthill, “Acoustic streaming," J. Sound Vib. 61, 391-418 (1978).

${ }^{18}$ Precisely speaking, this is the streaming motion of which the Reynolds number defined by $R s=U_{s} L / \nu$ is large compared with unity, where $U_{s}$ is a characteristic magnitude of the streaming motion, $L$ is a linear dimension of the system, and $\nu$ is the kinematic viscosity (cf. Ref. 17).

${ }^{19}$ L. D. Landau and E. M. Lifshitz, Fluid Mechanics (Pergamon, Oxford, 1987), 2nd ed., Sec. 10.

${ }^{20}$ G. K. Batchelor, An Introduction to Fluid Dynamics (Cambridge U.P., Cambridge, 1967), Chap. 5.

${ }^{21}$ A. Rizzi and L. E. Eriksson, "Computation of flow around wings based on the Euler equations," J. Fluid Mech. 148, 45-71 (1984).

${ }^{22}$ F. F. Grinstein, E. S. Oran, and J. P. Boris, "Numerical simulations of asymmetric mixing in planar shear flows," J. Fluid Mech. 165, 201-220 (1986).

${ }^{23}$ D. G. Crighton, "Acoustics as a branch of fluid mechanics," J. Fluid Mech. 106, 261-298 (1981).

${ }^{24}$ In the case that the separation occurs on a smooth boundary, the location of separation point depends on the Reynolds number and the numerical solutions to the Euler equations may not always provide the correct separation point due to the numerical viscosity.

${ }^{25} \mathrm{H}$. Hobæk, "On the acoustical near field of a circular piston source. Result from a numerical investigation," Scientific/Technical report No. 32 (1970), Department of Physics, University of Bergen.

${ }^{26}$ G. R. Harris, "Review of transient field theory for a baffled planar piston," J. Acoust. Soc. Am. 70, 10-20 (1981).

${ }^{27}$ J. Zemanek, "Beam behavior within the nearfield of a vibrating piston," J. Acoust. Soc. Am. 49, 181-191 (1971).

${ }^{28}$ C. Eckart, "Vortices and streams caused by sound waves," Phys. Rev. 73, 68-76 (1948). See also L. N. Liebermann, "The second viscosity of liquid," Phys. Rev. 75, 1415-1422 (1949).

${ }^{29}$ Y. Inoue, S. Ishii, and T. Okigami, "Propagation of finite amplitude sound waves radiated from a pulsating sphere,' J. Sound Vib. 106, 257-265 (1986).

${ }^{30}$ See Ref. 19, Sec. 114.

${ }^{31}$ This is the consequence of Kelvin's circulation theorem. Strictly speaking, however, once a shock is formed, the entropy cannot be regarded as uniform and hence one cannot apply Kelvin's theorem.

${ }^{32}$ A. Schoch, "Betrachtungen über das Schallfelt einer Kolbenmembran," Akust. Z. 6, 318-326 (1941). See also O. G. Kozina and G. I. Makarov, "Transient processes in the acoustic fields generated by a piston membrane of arbitrary shape," Sov. Phys. Acoust. 7, 39-43 (1961).

${ }^{33}$ I. S. Gradshteyn and I. M. Ryzhik, Table of Integrals, Series, and Products, Corrected and Enlarged Edition (Academic, San Diego, 1980), p. 1045 . 